\title{
Accurate force field of two-dimensional ferroelectrics from deep learning
}

\author{
Jing Wu, ${ }^{1,2, *}$ Liyi Bai, ${ }^{2,3, *}$ Jiawei Huang, ${ }^{4,2}$ Liyang Ma, ${ }^{1,2}$ Jian Liu, ${ }^{5,6}$ and Shi Liu ${ }^{2,3,7, f}$ \\ ${ }^{1}$ Fudan University, Shanghai 200433, China \\ ${ }^{2}$ School of Science, Westlake University, \\ Hangzhou, Zhejiang 310024, China \\ ${ }^{3}$ Institute of Natural Sciences, Westlake Institute for Advanced Study, \\ Hangzhou, Zhejiang 310024, China \\ ${ }^{4}$ Zhejiang University, Hangzhou Zhejiang 310058, P.R. China \\ ${ }^{5}$ Optics and Thermal Radiation Research Center, \\ Shandong University, Qingdao, Shandong 266237, China \\ ${ }^{6}$ School of Energy and Power Engineering, \\ Shandong University, Jinan, Shandong 250061, China \\ ${ }^{7}$ Key Laboratory for Quantum Materials of Zhejiang \\ Province, Hangzhou Zhejiang 310024, China
}

(Dated: November 1, 2021) 


\begin{abstract}
The discovery of two-dimensional (2D) ferroelectrics with switchable out-of-plane polarization such as monolayer $\alpha$ - $\operatorname{In}_{2} \mathrm{Se}_{3}$ offers a new avenue for ultrathin high-density ferroelectric-based nanoelectronics such as ferroelectric field effect transistors and memristors. The functionality of ferroelectrics depends critically on the dynamics of polarization switching in response to an external electric/stress field. Unlike the switching dynamics in bulk ferroelectrics that have been extensively studied, the mechanisms and dynamics of polarization switching in 2D remain largely unexplored. Molecular dynamics (MD) using classical force fields is a reliable and efficient method for largescale simulations of dynamical processes with atomic resolution. Here we developed a deep neural network-based force field of monolayer $\mathrm{In}_{2} \mathrm{Se}_{3}$ using a concurrent learning procedure that efficiently updates the first-principles-based training database. The model potential has accuracy comparable with density functional theory (DFT), capable of predicting a range of thermodynamic properties of $\mathrm{In}_{2} \mathrm{Se}_{3}$ polymorphs and lattice dynamics of ferroelectric $\mathrm{In}_{2} \mathrm{Se}_{3}$. Pertinent to the switching dynamics, the model potential also reproduces the DFT kinetic pathways of polarization reversal and $180^{\circ}$ domain wall motions. Moreover, isobaric-isothermal ensemble MD simulations predict a temperature-driven $\alpha \rightarrow \beta$ phase transition at the single-layer limit, as revealed by both local atomic displacement and Steinhardt's bond orientational order parameter $Q_{4}$. Our work paves the way for further research on the dynamics of ferroelectric $\alpha-\mathrm{In}_{2} \mathrm{Se}_{3}$ and related systems.
\end{abstract}

\footnotetext{
* These two authors contributed equally

$\dagger$ liushi@westlake.edu.cn
} 


\section{INTRODUCTIONS}

Ferroelectric materials with electrically addressable polarization have enabled various technological applications such as non-volatile memory, ferroelectric field effect transistors, sensors, and actuators [1 6]. The continuing demand for miniaturized electronics has inspired the seek of new ferroelectrics that exhibit reversible polarization at reduced dimensions. Thin films of ferroelectric perovskite such as $\mathrm{PbTiO}_{3}, \mathrm{BaTiO}_{3}$, and $\mathrm{BiFeO}_{3}$ have been the focus of numerous investigations, revealing a plethora of intriguing emergent phenomena that may hold the promise for next-generation electronics [7]. However, the depolarization effect in thin films often becomes detrimental when the film thickness is approaching the sub-100 nm regime [8]. The functional properties of ferroelectric thin films also depend sensitively on the lattice mismatch between the film and the underlying substrate as well as the growth quality of ferroelectric/metal interface [9, 10], causing scaling issue on an industry scale. The poor compatibility of perovskite ferroelectrics with silicon is another challenge hindering the development of ferroelectric memory technology [8].

Two-dimensional (2D) van der Waals (vdW) ferroelectrics with ferroelectric order in atom-thick layers are candidate materials addressing above issues because of their superior properties, i.e., uniform atomic thickness, the absence of surface dangling bonds, and the easy integration with other vdW layered materials. An increasing number of 2D ferroelectrics have been discovered by experiments or by first-principles calculations based on density functional theory (DFT) in the past decade [11]. Wu et al. proposed to functionalize graphene-based materials with hydroxyl groups to break the inversion symmetry in 2D [12]. Chang et al. reported robust in-plane ferroelectriciy in SnTe thin films with thickness down to one unit cell [13]. The phosphorene and phosphorene analogues such as GeS and GeSe monolayers were predicted to be multiferroics with coupled ferroelasticity and ferroelectricity at room temperatures [14]. In practice, 2D materials with out-of-plane polarization are desirable for high-density integration because of the easiness of lateral downscaling. Vertical ferroelectricity has been discovered in monolayer $\mathrm{CuInP}_{2} \mathrm{~S}_{6}$ [15], $\alpha$ - $\operatorname{In}_{2} \mathrm{Se}_{3}$ [16], distorted $1 T-\mathrm{MoS}_{2}$ [17, 18], $\mathrm{P}_{2} \mathrm{O}_{3}$ [19], and $\mathrm{MoTe}_{2}$ [20]. For vdW bilayers, the interlayer sliding is

recently recognized as a convenient route to out-of-plane polarization, applicable even to non-ferroelectric 2D materials such as $h$-BN and AlN [21-23].

Recently, $\mathrm{In}_{2} \mathrm{Se}_{3}$ has attracted significant attention for its diverse structural polymorphs 
and physical properties. Bulk $\operatorname{In}_{2} \mathrm{Se}_{3}$ can crystallize in several phases such as $\alpha(2 \mathrm{H}), \alpha(3 \mathrm{R})$, $\beta(3 \mathrm{R}), \gamma, \delta, \kappa$, where $\alpha$ and $\beta$ are layered vdW structures with $2 \mathrm{H}$ and $2 \mathrm{R}$ denoting different stacking sequences, and the other phases exhibit three-dimensional bonding networks [24]. The commonly accepted phase transformation of bulk $\operatorname{In}_{2} \mathrm{Se}_{3}$ is $\alpha \rightarrow \beta \rightarrow \gamma \rightarrow \delta$ with increasing temperature, though the exact phase transition temperature remains controversial [25 28]. Additionally, in-situ transmission electron microscopy characterizations suggested the formation of metastable phases, $\alpha^{\prime}, \beta^{\prime}$, and $\gamma^{\prime}$, during heating or cooling of $\alpha, \beta$, and $\gamma$ [24], respectively, highlighting the complexity of this indium selenides.

A typical monolayer in the layered phases of $\operatorname{In}_{2} \mathrm{Se}_{3}(\alpha$ and $\beta)$ is a covalently bonded quintuple layer $(\mathrm{QL})$ consisted of five atomic planes in the sequence of Se-In-Se-In-Se. Atomically thin $\mathrm{In}_{2} \mathrm{Se}_{3}$ can adopt a few phases with distinct lattice distortions, as illustrated in Figure 1 . Ding et al. systematically investigated all possible atomic configurations within a QL with DFT and found that the $\alpha$ - $\operatorname{In}_{2} \mathrm{Se}_{3}$ monolayer has the lowest energy and exhibits out-of-plane polarization $\left(P_{\mathrm{OP}}\right)$ and in-plane asymmetry. The presence of $P_{\mathrm{OP}}$ in multilayered $\alpha-\operatorname{In}_{2} \mathrm{Se}_{3}$ nanoflakes have been confirmed experimentally [29 33]. Notably, through second harmonic generation spectroscopy and piezoresponse force microscopy, Xiao et al. reported a robust out-of-plane ferroelectricity in $\alpha$ - $\mathrm{In}_{2} \mathrm{Se}_{3}$ crystals with thickness down to $3 \mathrm{~nm}$ and a high phase transition temperature $\left(T_{C}\right)$ of $700 \mathrm{~K}$ for $\alpha \rightarrow \beta$ structural transition [31]; Xue et al. reported ferroelectric hysteresis loops at the monolayer limit [30]. Structurally, the $\beta^{\prime}$ phase with in-plane polarization $\left(P_{\mathrm{IP}}\right)$ can be obtained by slightly displacing in-plane the central Se atoms of the nonpolar $\beta$ phase. Zheng et al. found that $\beta^{\prime}-\mathrm{In}_{2} \mathrm{Se}_{3}$ has stable in-plane ferroelectricity up to $473 \mathrm{~K}$ in both bulk crystals and thin layers down to $45 \mathrm{~nm}$ [34]. Zhang et al. discovered another phase at $77 \mathrm{~K}$ (named as $\beta^{\prime}$ in the original paper but $\beta_{3}^{\prime}$ in this work ) in $\mathrm{In}_{2} \mathrm{Se}_{3}$ with a thickness from more than 10 layers down to the monolayer [35]. We note that $\beta_{3}^{\prime}-\mathrm{In}_{2} \mathrm{Se}_{3}$ has canted in-plane dipoles resulting in a net in-plane polarization, a feature that is rare in bulk ferroelectrics and worthy of further investigations. Two metastable structure, $\beta_{1}^{\prime}$ and $\beta_{2}^{\prime}$, can be derived from the unstable phonon modes of $\beta-\mathrm{In}_{2} \mathrm{Se}_{3}$ at the center of the Brillouin zone followed by the break of in-plane threefold rotational symmetry. The in-plane lattice distortions in $\beta_{2}^{\prime}-\mathrm{In}_{2} \mathrm{Se}_{3}$ resemble those in the polar nanostripes of $\beta^{\prime}-\operatorname{In}_{2} \mathrm{Se}_{3}$ superstructures recently observed in experiments, where the periodic antiparallel ordering between neighboring nanostripes gives rise to $2 \mathrm{D}$ antiferroelectricity [36]. It is noted that $\alpha, \beta$ and $\beta^{\prime}$ phases have hexagonal symmetry whereas $\beta_{1}^{\prime}, \beta_{2}^{\prime}$, and $\beta_{3}^{\prime}$ phases are in the 
orthorhombic crystal family.

No different from their 3D counterparts, the functionality of $2 \mathrm{D}$ ferroelectrics depends critically on the response of the polarization to the external stimuli such as thermal, stress, and electric field. Apparently, a high $T_{C}$ is favored for everyday applications. Previous studies on various perovskite ferroelectrics showed that $T_{C}$ will decrease with reducing film thickness because of the stronger depolarization effect [37, 38]. In comparison, $\alpha-\operatorname{In}_{2} \operatorname{Se}_{3}$ nanoflakes exhibit an unusually high $T_{C}$ of $\approx 700 \mathrm{~K}$. The coercive field and the switching speed dictate the power consumption and writing speed of ferroelectric memory, respectively. The mechanisms and kinetics of ferroelectric switching in 3D ferroelectrics have been the focus of numerous experimental and theoretical studies. The commonly used models for domain switching are Kolmogorov-Avrami-Ishibashi (KAI) model [39-41], nucleation limited switching (NLS) model [42, 43], and homogeneous Landau switching model [44], the applicability of which depends largely on the sample dimensions. However, it remains questionable whether the domain switching models developed for 3D ferroelectrics are applicable at reduced dimensions. In particular, the domain wall in $2 \mathrm{D}$ ferroelectrics is essentially a 1D interface, which might have creep and depinning behavior fundamentally different from the 2D domain wall in bulk ferroelectrics [45]. Studying the dynamics of ferroelectric order in $2 \mathrm{D}$ is therefore both fundamentally and technologically important.

To observe and quantify the domain switching process in $2 \mathrm{D}$ ferroelectrics with sufficiently high spacial/time resolution remains a great experimental challenge. As a consensus, molecular dynamics (MD) is a suitable method to study the switching process at femtosecond and million atom scale [46, 47]. However, a force field that accurately describes the interatomic interactions is the prerequisite of running a MD simulation. Such force field is often not available for newly discovered materials. Since the discovery of graphene in 2004, a large number of 2D materials have been discovered. Nevertheless, only a handful of force fields of 2D materials have been developed, i.e., graphene [48 50], $\mathrm{MoS}_{2}$ [51], and $h$-BN [52, 53], most of which are based on the reactive empirical bond-order (REBO) potential [49]. Such scarcity reflects the difficulty of developing classical force fields for $2 \mathrm{D}$ materials. For a many-body potential such as REBO that uses a highly sophisticated energy function with a large number of parameters, the fitting process is often tedious. Moreover, there is no guaranty that the energy function developed for a specific class of material (e.g., hydrocarbon) is robust enough to represent the "true" interatomic potential of a different class 
(e.g., 2D ferroelectrics). Additionally, for a thermodynamic property, different force fields for the same material may predict drastically different values. For example, the thermal conductivity of graphene was calculated to be 870 [54], 709.2 [55], 350 [56], and $3000 \mathrm{~W}$ $\mathrm{mK}^{-1}$ [57, 58] using force fields of Terosff [59], AIREBO [48], REBO [49], and optimized Tersoff [50], respectively, presenting almost an order of magnitude variation.

Herein, using the Deep Potential Molecular Dynamics (DeePMD) method [60], we developed a deep neural network-based force field applicable to ferroelectric monolayer $\alpha-\operatorname{In}_{2} \mathrm{Se}_{3}$. The Deep Potential (DP) scheme represents the total energy as a sum of atomic energies, each parameterized with a deep neural network (DNN) taking atomic local environment as input (see details in ref. 60). The resultant DP model reproduces the DFT results for a range of thermodynamic properties of various phases of monolayer $\operatorname{In}_{2} \mathrm{Se}_{3}$. The DP energy barriers for a few polarization switching processes agree reasonably well with DFT values. Particularly, the temperature-driven ferroelectric-paraelectric phase transition of monolayer $\alpha$ - $\operatorname{In}_{2} \mathrm{Se}_{3}$ is captured by DPMD simulations, with a theoretical $T_{C} \approx 650 \mathrm{~K}$ matching with recent experimental value $(\approx 700 \mathrm{~K})$. Our work paves the way for further research on the dynamics of $2 \mathrm{D}$ ferroelectricity in monolayer $\alpha-\mathrm{In}_{2} \mathrm{Se}_{3}$ and related systems. We expect the current DP model can be systematically improved and extended, enabling large-scale simulations of interlayer sliding ferroelectricity and Moiré ferroelectricity of vdW multilayers.

\section{COMPUTATIONAL METHODS}

\section{A. Deep potential generator}

In this work, we employ the deep potential generator (DP-GEN) scheme [61] as implemented in DP-GEN package to develop the deep potential of $\mathrm{In}_{2} \mathrm{Se}_{3}$ with minimal human intervention. DP-GEN is a concurrent learning procedure with a closed loop consisted of three steps, exploration, labeling, and training, as shown in Figure 2. Additional details can be found in the original paper [61] and our recent work on developing a DP model of $\mathrm{HfO}_{2}$ [62]. In the training step, four models are trained with different initial values of hyperparameters of DNNs while using the same database of DFT energies and atomic forces. To gauge the necessity of DFT calculations for a configuration sampled from a DPMD run at the exploration step, the model deviation $\mathcal{E}$ is used, which is defined as the maximum 
standard deviation of the predictions of the atomic forces $\boldsymbol{F}_{i}$ from four different DP models,

$$
\mathcal{E}=\max _{i} \sqrt{\left\langle\left\|\boldsymbol{F}_{i}-\left\langle\boldsymbol{F}_{i}\right\rangle\right\|^{2}\right\rangle}
$$

where $i$ is the atomic index for atoms in a configuration and $\langle\ldots\rangle$ denotes the average of four DP models. We introduce two thresholds, $\mathcal{E}_{\text {lo }}$ and $\mathcal{E}_{\text {hi }}$; only configurations satisfying $\mathcal{E}_{\text {lo }}<\mathcal{E}<\mathcal{E}_{\text {hi }}$ are labeled for DFT calculations. That is, the model deviation serves as an indicator for importance sampling. For a configuration sampled from MD simulations at the exploration step, the four DP models in principle will exhibit nearly the same predictive accuracy $\left(\mathcal{E}<\mathcal{E}_{\mathrm{lo}}\right)$ if the configuration is well represented by the current training database. In contrary, a configuration with $\mathcal{E}>\mathcal{E}_{\text {lo }}$ indicates, heuristically, that it is "outside" the configuration space of the training database, the DFT energy and atomic forces of which will be added to the database for training in the next cycle. The introduction of $\mathcal{E}_{\text {hi }}$ is to handle the exceptions due to highly distorted configurations ( resulting from low-quality DP models especially in the first few cycles of DP-GEN) or unconverged DFT calculations. When all sampled configurations have $\mathcal{E}<\mathcal{E}_{\text {lo }}$, the ensemble of DP models is considered converged. Here we set $\mathcal{E}_{\mathrm{lo}}=0.12 \mathrm{eV} / \AA$ and $\mathcal{E}_{\mathrm{hi}}=0.25 \mathrm{eV} / \AA$.

The smooth version of the DP model with an inner cutoff of $1 \AA$ and an outer cutoff of $6 \AA$ was adopted [63] in this study, and the DeePMD-kit package [64] was used for training. The embedding network has the ResNet-like architecture with a size of $(25,50,100)$. The fitting network comprises three layers, each containing 240 nodes, with the loss function defined as

$$
L\left(p_{\epsilon}, p_{f}, p_{\xi}\right)=p_{\epsilon} \Delta \epsilon^{2}+\frac{p_{f}}{3 N} \sum_{i}\left|\Delta \boldsymbol{F}_{i}\right|^{2}+\frac{p_{\xi}}{9}\|\Delta \xi\|^{2}
$$

where $\Delta$ denotes the difference between the DP results and the training data, $N$ is the number of atoms, $\epsilon$ is the energy per atom, $\boldsymbol{F}_{i}$ is the atomic force of atom $i$, and $\xi$ is the virial tensor divided by $N$. The parameters, $p_{\epsilon}, p_{f}$, and $p_{\xi}$, essentially tune the weights of energy, force, and stress information used in training, and can be changed even during the learning process [64]. Here we increase both $p_{\epsilon}$ and $p_{\xi}$ from 0.02 at the beginning to 1 as the learning ends, while $p_{f}$ decreases from 1000 to 1 . The number of batch and step of learning rate decay are set to 4000000 and 20000, respectively. 


\section{B. Initial training database and exploration protocol}

The DP-GEN scheme allows for automatic, iterative, and efficient updates of the training database, making the force field development less impacted by the construction of the initial training database. Nevertheless, a carefully designed initial database with diverse configurations could speed up the reach of model convergence. The initial training database contains ground-state structures and configurations with random atomic/strain perturbations of bulk polymorphs (space group: $P \overline{3} m 1, R 3 m$, and $P 6_{1}$ ) and monolayer of different

phases including $\alpha, \beta^{\prime}, \beta_{1}^{\prime}, \beta_{2}^{\prime}$, and $\beta$ phases. We use a 20-atom slab model to represent the monolayer system and a 30-atom supercell to model the bulk. DFT calculations are carried out with the Vienna Ab initio Simulation (VASP) package [65, 66], employing the projected augmented wave (PAW) method [67, 68] and the generalized gradient approximation of Perdew-Burke-Ernzerhof (PBE) [69] type for the exchange-correlation functional as most of previous first-principles DFT studies of monolayer $\operatorname{In}_{2} \mathrm{Se}_{3}$ used PBE. A DP model trained with a PBE-based database allows us to directly compare DP predictions with previous DFT results. The main focus of this work is to develop an accurate model potential for monolayer $\mathrm{In}_{2} \mathrm{Se}_{3}$. For this reason, the vdW correction was not employed as it is likely not important for intralayer interactions dominated by covalent bonds.

An energy cutoff of $700 \mathrm{eV}$ and a $k$-point spacing of $0.3 \AA^{-1}$ (corresponding to a $4 \times 4 \times 1 k$ point mesh for a 20-atom slab model) are sufficient to converge the energy and atomic force. At the exploration step, the configuration space is sampled by running NPT simulations at various temperatures (from 300 to $1200 \mathrm{~K}$ ) at 0 and $1 \mathrm{kBar}$, respectively.

\section{MD simulations of phase transition}

The converged DP model of $\mathrm{In}_{2} \mathrm{Se}_{3}$ is used to investigate the temperature-driven phase transition of monolayer $\alpha-\mathrm{In}_{2} \mathrm{Se}_{3}$ by running NPT MD simulations from $300 \mathrm{~K}$ to $1200 \mathrm{~K}$ with a temperature interval of $50 \mathrm{~K}$. Similar to our previous study on $\mathrm{HfO}_{2}$ [62], we found that the value of $T_{C}$ from MD exhibits supercell size effect. We thus used a sufficiently large 8000 -atom supercell consisted of $40 \times 40 \times 1$ unit cells to obtain the converged $T_{C}$. The MD trajectories are propagated using the velocity Verlet algorithm [70] and a time step of 1 fs, with temperature and pressure controlled by Nosé-Hoover thermostat and Parrinello- 
Rahman barostat, respectively, as implemented in LAMMPS [71]. At each temperature, an equilibrium run of at least $10 \mathrm{ps}$ is performed followed by a 60 ps production run that samples configurations for property analysis. At temperatures close to $T_{C}$, the production run is 500 ps to ensure enough sampling to obtain statistically reliable values of thermodynamics properties.

\section{RESULTS}

\section{A. Fitting performance of DP model}

The final training database records 22600 monolayer configurations and 2163 bulk structures. The model deviation $\mathcal{E}$ indeed serves as an efficient indicator of importance sampling. The convergence was reached after 20 iterations during which more than 8 million configurations were sampled while only 7124 (0.09\%) configurations were deemed important for DFT calculations, substantially reducing the computational cost associated with DFT calculations. Moreover, the automatic and iterative updates of the training database on the fly greatly alleviate the burden and reduce the bias of human interventions.

Figure 3 compares the energy per atom and atomic forces predicted by DFT and DP for all the structures, with the insets illustrating the absolute error distributions. The excellent representability of the DNN-based potential is evident from the small mean absolute error (MAE) of just $5.72 \mathrm{meV} /$ atom for nearly 25000 configurations. Additionally, the training database contains a set of configurations that construct a 2D potential energy surface for the in-plane sliding of the central Se sublayer within the QL. The DFT and DP energy maps reported in Figure 4 show remarkable agreement with a MAE of $2.68 \mathrm{meV} /$ atom. The capability to faithfully reproduce the energies and atomic forces of a diverse set of configurations gives the trained DP model a firm basis to accurately predict thermodynamic and kinetic properties of $\operatorname{In}_{2} \mathrm{Se}_{3}$.

\section{B. Predictions of static properties of $\operatorname{In}_{2} \mathrm{Se}_{3}$}

Unlike a supervised learning task that deals with a fixed data distribution from which the validation dataset can be drawn to check generalization and overfitting problems, the accuracy of a DP model is better confirmed by comparing DP predictions with DFT and/or 
known experimental results not included in the training database [62]. We first check the predictive performance of the DP model by computing various thermodynamic properties. Table I compares the DFT and DP energies of different phases of monolayer $\mathrm{In}_{2} \mathrm{Se}_{3}$, demonstrating an excellent agreement. The DP model predicts the correct energetic ordering $E(\alpha)<E\left(\beta_{3}^{\prime}\right)<E\left(\beta_{1}^{\prime}\right)<E\left(\beta^{\prime}\right)<E\left(\beta_{2}^{\prime}\right)<E(\beta)$. In particular, the energy differences between those $\beta$-derived phases are within $2 \mathrm{meV} /$ atom, all reproduced by the DP model. Figure 5 presents the DP and DFT energy-strain plots for monolayers subjected to equibiaxial deformations. The DP model achieves a satisfactory accuracy in this test as well: the DFT and DP curves are nearly overlapping with each other over a wide strain range $( \pm 6 \%)$. We note that the experimentally observed $\beta_{3}^{\prime}$ phase, though not included in the training database, is described reasonably well by the DP model, hinting at some level of transferability of the DP model.

Accurate prediction of the phonon dispersion relationship is essential for quantitative evaluations of thermodynamic properties at finite temperatures. The lattice constant of $\alpha$ $\mathrm{In}_{2} \mathrm{Se}_{3}$ optimized with the DP model is $a=4.101 \AA$, within $1 \%$ of the DFT value of $4.063 \AA$. We then compute the phonon spectra of $\alpha-\operatorname{In}_{2} \mathrm{Se}_{3}$ with DFT and DP, respectively, using the frozen phonon approach as implemented in Phonopy [72]. As shown in Figure 6, the DP phonon spectrum of monolayer $\alpha-\mathrm{In}_{2} \mathrm{Se}_{3}$ show a decent agreement with DFT spectrum. It is often challenging to reproduce first-principles phonon dispersion relationships for a classical force field because of the demanding requirement to accurately fit the second-order derivatives of the potential energy surface. The confirmed accuracy of the DP model for predicting lattice dynamics highlights the robustness of the DP-GEN scheme that does not explicitly include perturbed structures generated within the frozen phonon approximation for phonon spectrum calculations. It is expected that the DP model can be further improved if all the perturbed supercells are included in the training database.

\section{Domain switching}

In order to use MD to study polarization reversal at finite temperatures, it is necessary for the force field to accurately reproduce the DFT kinetic barriers of representative processes at $0 \mathrm{~K}$. Structurally, the out-of-plane polarization of $\alpha-\operatorname{In}_{2} \mathrm{Se}_{3}$ is due to the displacement of the central Se layer with respect to top and bottom In-Se layers. Ding et al. identified 
two polarization reversal pathways with the nudged elastic band (NEB) method: the direct shifting of the central Se layer and a three-step concerted motion of upper Se-In-Se layers [16], as shown in Figure 7. We determined the the minimum energy paths (MEPs) using the NEB technique implemented in the USPEX [73-75] code based on the switching trajectories proposed in ref.16. It is noted that the DFT calculations of energy and force required in the NEB method were performed using the same settings (e.g., cutoff energy and $k$-point density) as those at the labeling step of the DP-GEN scheme. The energies of configurations along the MEPs were then evaluated with the DP model. As shown in Figure 7, the DFT and DP energy profiles of the two polarization reversal pathways are nearly identical with a small MAE of $1.68 \mathrm{meV} /$ atom. In particular, the reproduction of the DFT energy profile for the complicated three-step switching process (Fig. 7(b)) that involves highly distorted intermediate structures manifests the quantitative nature of the DP model.

For bulk ferroelectrics, it is well established that the electric field-driven domain wall motion plays a critical role in ferroelectric switching. It is thus essential for the DP model to reproduce the energy barrier of lateral domain wall motion. Because of the in-plane lattice asymmetry, there are four different types of $180^{\circ}$ domain walls in monolayer $\alpha$ - $\mathrm{In}_{2} \mathrm{Se}_{3}$. The initial pathways of $180^{\circ}$ domain wall motions were taken from ref.[16 and were re-optimized using a higher planewave cutoff energy and more intermediate images. As shown in Fig. 8 , the DP energy profiles again agree with DFT results for all four pathways.

\section{Phase transitions}

Our main purpose is to obtain an accurate force field for MD simulations of monolayer $\mathrm{In}_{2} \mathrm{Se}_{3}$. We simulate the temperature-driven phase transition starting with the ferroelectric $\alpha$ phase using the DP model and a 8000-atom supercell. The out-of-plane displacement $\left(D_{z}\right)$ of

the central Se atoms with respect to the geometric center of the monolayer is used to measure the local symmetry breaking. Figure 9 (a) shows the evolution of the probability distribution of $D_{z}$ with increasing temperature. At each temperature, the distribution of $D_{z}$ can be fitted with a single Gaussian. The peak position shifts towards lower values and sharply decreases to zero when the temperature changes from $600 \mathrm{~K}$ to $650 \mathrm{~K}$ (see Fig. 9(a) inset), which reveals a ferroelectric-to-paraelectric $(\alpha \rightarrow \beta)$ phase transition (see the trajectory movies of $D_{z}$ maps in a public repository) [76]. The value of $T_{C}$ from DPMD simulations is $\approx 650 \mathrm{~K}$, 
agreeing with previous first-principles MD studies $(\approx 650 \mathrm{~K})$ [77] and experimental value of $700 \mathrm{~K}$ in 3-nm-thick $\alpha$ - $\operatorname{In}_{2} \mathrm{Se}_{3}$ crystals [31]. Our large-scale MD simulations suggest that the $\alpha \rightarrow \beta$ transition is mostly of the displacive character [78, 79]. We also plot the 2D maps of $D_{z}$ for instantaneous configurations sampled at different temperatures in Fig. 9(b). Consistent with the probability distribution of $D_{z}$, the values of $D_{z}$ also show considerable spacial variations when $T>T_{C}$.

The Steinhardt's bond orientational order parameters $\left(Q_{l}\right)$ [80, 81] are most commonly used to distinguish different crystalline phases and clusters of particles in 3D. The $Q_{l}$ at site $i$ is calculated according to

$$
\begin{gathered}
Q_{l}=\sqrt{\left(\frac{4 \pi}{2 l+1}\right) \sum_{m=-l}^{m=l} \bar{Y}_{l m} \bar{Y}_{l m}^{*}} \\
\bar{Y}_{l m}=\frac{1}{N_{b}(i)} \sum_{j \in N_{b}(i)} Y_{l m}\left(\theta\left(r_{i j}\right), \phi\left(r_{i j}\right)\right)
\end{gathered}
$$

where $l$ is the order of symmetry, $\bar{Y}_{l m}$ is the average of the spherical harmonics $Y_{l m}\left(\theta\left(r_{i j}\right), \phi\left(r_{i j}\right)\right)$ with $\theta\left(r_{i j}\right)$ and $\phi\left(r_{i j}\right)$ being the azimuthal and polar angles of the bond pointing from particle $i$ to particle $j$, respectively, and $N_{b}(i)$ is the number of neighbors of atom $i$. It is easy to see that $Q_{l}$ is rotational invariant and non-negative. Table II reports the layer-resolved $Q_{4}$ and $Q_{6}$ values of Se atoms in $\alpha$ and $\beta$ phases using DFT optimized structures. It shows that Se atoms have distinct $Q_{4}$ and $Q_{6}$ values in these two phases. In particular, $Q_{4}$ of Se atoms of the middle layer in the $\beta$ phase (0.082) is almost twice the value than that in the $\alpha$ phase (0.049). Because of the out-of-plane polarization, there are three symmetrically unique Se atoms in the $\alpha$ phase, corresponding to different $Q_{4}$ and $Q_{6}$ values.

We calculate $Q_{4}$ and $Q_{6}$ of all the Se atoms for configurations sampled at different temperatures and present their violin plots in Fig. 10(a). The density curves of $Q_{4}$ at $T<T_{C}$ exhibit two peaks, with the higher value peak $\left(\Lambda_{h}\right)$ corresponding to surface Se atoms and the lower value peak $\left(\Lambda_{l}\right)$ coming from central Se atoms, and the value of $\Lambda_{l}$ increases with temperature. At $T>T_{C}$, the density curves become single peaked. Overall, $Q_{4}$ serves as a good indicator of local atomic environment and $\alpha \rightarrow \beta$ phase transition. The density curves of $Q_{6}$ change from distributions possessing multiple peaks below $T_{C}$ to unimodal distributions above $T_{C}$. Finally, we present $2 \mathrm{D}$ maps of $Q_{4}$ for Se atoms in the middle sublayer for instantaneous configurations sampled at selected temperatures (Fig. 10(b)). Similar to the 
2D $D_{z}$ maps shown in Fig. 9 (b), $Q_{4}$ maps also reveal substantial structural heterogeneity in space particularly at temperatures above $T_{C}$.

\section{CONCLUSIONS}

Using the concurrent learning scheme, we developed a DP model for monolayer $\mathrm{In}_{2} \mathrm{Se}_{3}$. The model potential exhibits accuracy comparable with first-principles methods, as confirmed by the excellent agreement between DFT and DP results for various thermodynamic properties of $\operatorname{In}_{2} \mathrm{Se}_{3}$ polymorphs and kinetic pathways of polarization reveal and $180^{\circ}$ domain wall motions in $\alpha-\mathrm{In}_{2} \mathrm{Se}_{3}$. The constant-temperature constant-pressure MD simulations predict a temperature-driven $\alpha \rightarrow \beta$ phase transition at the single-layer limit. Both local atomic displacement and Steinhardt's bond orientational order parameter $Q_{4}$ are used to characterize the phase transition, revealing a displacive feature. The DP model developed in this work could be a starting point for research of dynamical processes in $\alpha$ - $\operatorname{In}_{2} \mathrm{Se}_{3}$-based nanoelectronics. Our work demonstrates the robustness of DP for 2D materials. Following a similar protocol we suggested in our previous work [62], we make our final training database and hyperparameters available through a public repository [82], which can be used for training a new DP model or any other ML-based force field based on desired exchange-correlation functionals such as SCAN that has demonstrated accuracy for solid-state materials [83 85]. We expect the current DP model can be systematically improved and extended, enabling large-scale simulations of interlayer sliding ferroelectricity and Moiré ferroelectricity of vdW

multilayers. Specifically, we can add new configurations of bilayer $\operatorname{In}_{2} \mathrm{Se}_{3}$ of different phases to the current training database and set up exploration runs of various bilayer systems at different temperatures to generate new configurations in the DP-GEN scheme. After a converged training with a database containing diverse configurations of layered systems, we believe the DP model, thanks to the ability of the deep neural network to faithfully represent complex energy landscape, can accurately describe the subtle changes in energy and atomic forces during the slide and rotation of two monolayers, essential for the modeling of interlayer sliding ferroelectricity and Moiré ferroelectricity. 


\section{ACKNOWLEDGMENTS}

J.W.,L.B.,J.H., L.M., and S.L.acknowledge the support from Westlake Education Foundation, Westlake Multidisciplinary Research Initiative Center, and the National Natural Science Foundation of China (52002335). The computational resources were provided by Westlake HPC Center. The authors thank Dr. Wenjun Ding for sharing the trajectories of polarization reversal and domain wall motions in moonolayer $\alpha-\operatorname{In}_{2} \mathrm{Se}_{3}$. The authors also thank Zhe Wang and Dr. Wenguang Zhu for providing the structure file of $\beta_{3}^{\prime}-\operatorname{In}_{2} \mathrm{Se}_{3}$.

[1] V. Garcia, S. Fusil, K. Bouzehouane, S. Enouz-Vedrenne, N. D. Mathur, A. Barthélémy, and M. Bibes, Giant tunnel electroresistance for non-destructive readout of ferroelectric states, Nature 460, 81 (2009).

[2] H. Lu, C.-W. Bark, D. E. de los Ojos, J. Alcala, C. B. Eom, G. Catalan, and A. Gruverman, Mechanical writing of ferroelectric polarization, Science 336, 59 (2012).

[3] S. Wan, Y. Li, W. Li, X. Mao, C. Wang, C. Chen, J. Dong, A. Nie, J. Xiang, Z. Liu, W. Zhu, and H. Zeng, Nonvolatile ferroelectric memory effect in ultrathin $\alpha-\operatorname{In}_{2} \mathrm{Se}_{3}, \mathrm{Adv}$. Funct. Mater. 29, 1808606 (2019).

[4] L. Liao, H. J. Fan, B. Yan, Z. Zhang, L. L. Chen, B. S. Li, G. Z. Xing, Z. X. Shen, T. Wu, X. W. Sun, J. Wang, and T. Yu, Ferroelectric transistors with nanowire channel: Toward nonvolatile memory applications, ACS Nano 3, 700 (2009).

[5] M. Si, C.-J. Su, C. Jiang, N. J. Conrad, H. Zhou, K. D. Maize, G. Qiu, C.-T. Wu, A. Shakouri, M. A. Alam, and P. D. Ye, Steep-slope hysteresis-free negative capacitance $\mathrm{MoS}_{2}$ transistors, Nat. Nanotechnol. 13, 24 (2017)

[6] X. Wang, J. Zhou, J. Song, J. Liu, N. Xu, and Z. L. Wang, Piezoelectric field effect transistor and nanoforce sensor based on a single ZnO nanowire, Nano Lett. 6, 2768 (2006).

[7] M. Kinyanjui, Y. Lu, N. Gauquelin, M. Wu, A. Frano, P. Wochner, M. Reehuis, G. Christiani, G. Logvenov, H.-U. Habermeier, et al., Lattice distortions and octahedral rotations in epitaxially strained $\mathrm{LaNiO}_{3} / \mathrm{LaAlO}_{3}$ superlattices, Appl. Phys. Lett. 104, 221909 (2014).

[8] C.-U. Pinnow and T. Mikolajick, Material aspects in emerging nonvolatile memories, J. Electrochem. Soc. 151, K13 (2004). 
[9] Y. W. Yin, J. D. Burton, Y.-M. Kim, A. Y. Borisevich, S. J. Pennycook, S. M. Yang, T. W. Noh, A. Gruverman, X. G. Li, E. Y. Tsymbal, and Q. Li, Enhanced tunnelling electroresistance effect due to a ferroelectrically induced phase transition at a magnetic complex oxide interface, Nat. Mater. 12, 397 (2013).

[10] L. W. Martin and A. M. Rappe, Thin-film ferroelectric materials and their applications, Nat. Rev. Mater. 2, 16087 (2016).

[11] M. Wu, Two-dimensional van der waals ferroelectrics: Scientific and technological opportunities, ACS Nano 15, 9229 (2021).

[12] M. Wu, J. D. Burton, E. Y. Tsymbal, X. C. Zeng, and P. Jena, Hydroxyl-decorated graphene systems as candidates for organic metal-free ferroelectrics, multiferroics, and high-performance proton battery cathode materials, Phys. Rev. B 87, 081406 (2013).

[13] K. Chang, J. Liu, H. Lin, N. Wang, K. Zhao, A. Zhang, F. Jin, Y. Zhong, X. Hu, W. Duan, Q. Zhang, L. Fu, Q.-K. Xue, X. Chen, and S.-H. Ji, Discovery of robust in-plane ferroelectricity in atomic-thick SnTe, Science 353, 274 (2016).

[14] M. Wu and X. C. Zeng, Intrinsic ferroelasticity and/or multiferroicity in two-dimensional phosphorene and phosphorene analogues, Nano Lett. 16, 3236 (2016).

[15] A. Belianinov, Q. He, A. Dziaugys, P. Maksymovych, E. Eliseev, A. Borisevich, A. Morozovska, J. Banys, Y. Vysochanskii, and S. V. Kalinin, $\mathrm{CuInP}_{2} \mathrm{~S}_{6}$ room temperature layered ferroelectric, Nano Lett. 15, 3808 (2015).

[16] W. Ding, J. Zhu, Z. Wang, Y. Gao, D. Xiao, Y. Gu, Z. Zhang, and W. Zhu, Prediction of intrinsic two-dimensional ferroelectrics in $\mathrm{In}_{2} \mathrm{Se}_{3}$ and other $\mathrm{III}_{2}-\mathrm{VI}_{3}$ van der waals materials, Nat. Commun. 8, 14956 (2017).

[17] S. N. Shirodkar and U. V. Waghmare, Emergence of ferroelectricity at a metal-semiconductor transition in a $1 T$ monolayer of $\mathrm{MoS}_{2}$, Phys. Rev. Lett. 112, 157601 (2014).

[18] E. Bruyer, D. Di Sante, P. Barone, A. Stroppa, M.-H. Whangbo, and S. Picozzi, Possibility of combining ferroelectricity and rashba-like spin splitting in monolayers of the $1 T$-type transition-metal dichalcogenides $M X_{2}(M=\mathrm{Mo}, \mathrm{W} ; X=\mathrm{S}, \mathrm{Se}, \mathrm{Te})$, Phys. Rev. B 94, 195402 (2016).

[19] W. Luo and H. Xiang, Two-dimensional phosphorus oxides as energy and information materials, Angew. Chem. Int. Ed. 55, 8575 (2016). 
[20] S. Yuan, X. Luo, H. L. Chan, C. Xiao, Y. Dai, M. Xie, and J. Hao, Room-temperature ferroelectricity in $\mathrm{MoTe}_{2}$ down to the atomic monolayer limit, Nat. Commun. 10, 1775 (2019).

[21] L. Li and M. Wu, Binary compound bilayer and multilayer with vertical polarizations: Twodimensional ferroelectrics, multiferroics, and nanogenerators, ACS Nano 11, 6382 (2017)

[22] T. Zhang, Y. Liang, X. Xu, B. Huang, Y. Dai, and Y. Ma, Ferroelastic-ferroelectric multiferroics in a bilayer lattice, Phys. Rev. B 103, 165420 (2021).

[23] K. Yasuda, X. Wang, K. Watanabe, T. Taniguchi, and P. Jarillo-Herrero, Stacking-engineered ferroelectricity in bilayer boron nitride, Science 372, 1458 (2021).

[24] G. Han, Z.-G. Chen, J. Drennan, and J. Zou, Indium selenides: Structural characteristics, synthesis and their thermoelectric performances, Small 10, 2747 (2014).

[25] S. Popović, A. Tonejc, B. Gržeta-Plenković, B. Čelustka, and R. Trojko, Revised and new crystal data for indium selenides, J. Appl. Crystallogr. 12, 416 (1979).

[26] K. Osamura, Y. Murakami, and Y. Tomiie, Crystal structures of $\alpha$-and $\beta$-indium selenide, $\mathrm{In}_{2} \mathrm{Se}_{3}$, J. Phys. Soc. Japan 21, 1848 (1966).

[27] H. Lutz, M. Fischer, H.-P. Baldus, and R. Blachnik, Zur polymorphie des $\mathrm{In}_{2} \mathrm{Se}_{3}$, J. lesscommon met. 143, $83(1988)$.

[28] M. Küpers, P. M. Konze, A. Meledin, J. Mayer, U. Englert, M. Wuttig, and R. Dronskowski, Controlled crystal growth of indium selenide, $\mathrm{In}_{2} \mathrm{Se}_{3}$, and the crystal structures of $\alpha-\mathrm{In}_{2} \mathrm{Se}_{3}$, Inorg. Chem. 57, 11775 (2018),

[29] C. Cui, W.-J. Hu, X. Yan, C. Addiego, W. Gao, Y. Wang, Z. Wang, L. Li, Y. Cheng, P. Li, X. Zhang, H. N. Alshareef, T. Wu, W. Zhu, X. Pan, and L.-J. Li, Intercorrelated in-plane and out-of-plane ferroelectricity in ultrathin two-dimensional layered semiconductor $\mathrm{In}_{2} \mathrm{Se}_{3}, \mathrm{Nano}$ Lett. 18, 1253 (2018).

[30] F. Xue, J. Zhang, W. Hu, W.-T. Hsu, A. Han, S.-F. Leung, J.-K. Huang, Y. Wan, S. Liu, J. Zhang, J.-H. He, W.-H. Chang, Z. L. Wang, X. Zhang, and L.-J. Li, Multidirection piezoelectricity in mono- and multilayered hexagonal $\alpha-\operatorname{In}_{2} \mathrm{Se}_{3}$, ACS Nano 12, 4976 (2018).

[31] J. Xiao, H. Zhu, Y. Wang, W. Feng, Y. Hu, A. Dasgupta, Y. Han, Y. Wang, D. A. Muller, L. W. Martin, P. Hu, and X. Zhang, Intrinsic two-dimensional ferroelectricity with dipole locking, Phys. Rev. Lett. 120, 227601 (2018).

[32] S. M. Poh, S. J. R. Tan, H. Wang, P. Song, I. H. Abidi, X. Zhao, J. Dan, J. Chen, Z. Luo, S. J. Pennycook, A. H. C. Neto, and K. P. Loh, Molecular-beam epitaxy of two-dimensional 
$\mathrm{In}_{2} \mathrm{Se}_{3}$ and its giant electroresistance switching in ferroresistive memory junction, Nano Lett. 18, 6340 (2018).

[33] F. Xue, W. Hu, K.-C. Lee, L.-S. Lu, J. Zhang, H.-L. Tang, A. Han, W.-T. Hsu, S. Tu, W.-H. Chang, C.-H. Lien, J.-H. He, Z. Zhang, L.-J. Li, and X. Zhang, Room-temperature ferroelectricity in hexagonally layered $\alpha-\mathrm{In}_{2} \mathrm{Se}_{3}$ nanoflakes down to the monolayer limit, Adv. Funct. Mater. 28, 1803738 (2018),

[34] C. Zheng, L. Yu, L. Zhu, J. L. Collins, D. Kim, Y. Lou, C. Xu, M. Li, Z. Wei, Y. Zhang, M. T. Edmonds, S. Li, J. Seidel, Y. Zhu, J. Z. Liu, W.-X. Tang, and M. S. Fuhrer, Room temperature in-plane ferroelectricity in van der waals $\operatorname{In}_{2} \mathrm{Se}_{3}$, Sci. Adv. 4, eaar7720 (2018).

[35] F. Zhang, Z. Wang, J. Dong, A. Nie, J. Xiang, W. Zhu, Z. Liu, and C. Tao, Atomic-scale observation of reversible thermally driven phase transformation in $2 \mathrm{D} \mathrm{In}_{2} \mathrm{Se}_{3}$, ACS Nano 13, 8004 (2019).

[36] C. Xu, Y. Chen, X. Cai, A. Meingast, X. Guo, F. Wang, Z. Lin, T. W. Lo, C. Maunders, S. Lazar, N. Wang, D. Lei, Y. Chai, T. Zhai, X. Luo, and Y. Zhu, Two-dimensional antiferroelectricity in nanostripe-ordered $\mathrm{In}_{2} \mathrm{Se}_{3}$, Phys. Rev. Lett. 125, 047601 (2020).

[37] S. K. Streiffer, J. A. Eastman, D. D. Fong, C. Thompson, A. Munkholm, M. V. Ramana Murty, O. Auciello, G. R. Bai, and G. B. Stephenson, Observation of nanoscale $180^{\circ}$ stripe domains in ferroelectric $\mathrm{PbTiO}_{3}$ thin films, Phys. Rev. Lett. 89, 067601 (2002).

[38] M. Glinchuk, E. Eliseev, and V. Stephanovich, The depolarization field effect on the thin ferroelectric films properties, Physica B Condens. Matter 322, 356 (2002).

[39] A. Kolmogoroff, On the statistical theory of metal crystallization, Izv. Akad. Nauk SSSR Ser. Mat. 3, 335 (1937).

[40] M. Avrami, Kinetics of phase change. I general theory, J. Chem. Phys. 7, 1103 (1939).

[41] Y. Ishibashi and Y. Takagi, Note on ferroelectric domain switching, J. Phys. Soc. Japan 31, 506 (1971).

[42] J. Jo, H. Han, J.-G. Yoon, T. Song, S.-H. Kim, and T. Noh, Domain switching kinetics in disordered ferroelectric thin films, Phys. Rev. Lett. 99, 267602 (2007).

[43] A. K. Tagantsev, I. Stolichnov, N. Setter, J. S. Cross, and M. Tsukada, Non-kolmogorovavrami switching kinetics in ferroelectric thin films, Phys. Rev. B 66, 214109 (2002).

[44] M. Hoffmann, F. P. G. Fengler, M. Herzig, T. Mittmann, B. Max, U. Schroeder, R. Negrea, P. Lucian, S. Slesazeck, and T. Mikolajick, Unveiling the double-well energy landscape in a 
ferroelectric layer, Naure 565, 464 (2019).

[45] P. Chauve, T. Giamarchi, and P. Le Doussal, Creep and depinning in disordered media, Phys. Rev. B 62, 6241 (2000),

[46] S. Liu, I. Grinberg, and A. M. Rappe, Intrinsic ferroelectric switching from first principles, Naure 534, 360 (2016).

[47] H. Takenaka, I. Grinberg, S. Liu, and A. M. Rappe, Slush-like polar structures in single-crystal relaxors, Naure 546, 391 (2017).

[48] S. J. Stuart, A. B. Tutein, and J. A. Harrison, A reactive potential for hydrocarbons with intermolecular interactions, J. Chem. Phys. 112, 6472 (2000).

[49] D. W. Brenner, O. A. Shenderova, J. A. Harrison, S. J. Stuart, B. Ni, and S. B. Sinnott, A second-generation reactive empirical bond order (REBO) potential energy expression for hydrocarbons, J. Phys. Condens. Matter 14, 783 (2002).

[50] L. Lindsay and D. A. Broido, Optimized tersoff and brenner empirical potential parameters for lattice dynamics and phonon thermal transport in carbon nanotubes and graphene, Phys. Rev. B 81, 205441 (2010).

[51] T. Liang, S. R. Phillpot, and S. B. Sinnott, Parametrization of a reactive many-body potential for Mo-S systems, Phys. Rev. B 79, 245110 (2009).

[52] B. Mortazavi, L. F. C. Pereira, J.-W. Jiang, and T. Rabczuk, Modelling heat conduction in polycrystalline hexagonal boron-nitride films, Sci. Rep. 5, 13228 (2015).

[53] A. G. Rajan, M. S. Strano, and D. Blankschtein, Ab initio molecular dynamics and lattice dynamics-based force field for modeling hexagonal boron nitride in mechanical and interfacial applications, J. Phys. Chem. Lett. 9, 1584 (2018).

[54] Z. Wei, Z. Ni, K. Bi, M. Chen, and Y. Chen, In-plane lattice thermal conductivities of multilayer graphene films, Carbon 49, 2653 (2011).

[55] Y. Hong, M. G. Ju, J. Zhang, and X. C. Zeng, Phonon thermal transport in a graphene/MoSe2 van der waals heterobilayer, Phys. Chem. Chem. Phys. 20, 2637 (2018).

[56] J. A. Thomas, R. M. Iutzi, and A. J. H. McGaughey, Thermal conductivity and phonon transport in empty and water-filled carbon nanotubes, Phys. Rev. B 81, 045413 (2010).

[57] B. Mortazavi and T. Rabczuk, Multiscale modeling of heat conduction in graphene laminates, Carbon 85, 1 (2015). 
[58] Z. Fan, L. F. C. Pereira, P. Hirvonen, M. M. Ervasti, K. R. Elder, D. Donadio, T. Ala-Nissila, and A. Harju, Thermal conductivity decomposition in two-dimensional materials: Application to graphene, Phys. Rev. B 95, 144309 (2017).

[59] J. Tersoff, Modeling solid-state chemistry: Interatomic potentials for multicomponent systems, Phys. Rev. B 39, 5566 (1989).

[60] Y. Zhang, H. Wang, W. Chen, J. Zeng, L. Zhang, H. Wang, and W. E, DP-GEN: A concurrent learning platform for the generation of reliable deep learning based potential energy models, Comput. Phys. Commun. 253, 107206 (2020).

[61] L. Zhang, D.-Y. Lin, H. Wang, R. Car, and W. E, Active learning of uniformly accurate interatomic potentials for materials simulation, Phys. Rev. Materials 3, 023804 (2019).

[62] J. Wu, Y. Zhang, L. Zhang, and S. Liu, Deep learning of accurate force field of ferroelectric $\mathrm{HfO}_{2}$, Phys. Rev. B 103, 024108 (2021).

[63] L. Zhang, J. Han, H. Wang, W. A. Saidi, R. Car, and E. Weinan, End-to-end symmetry preserving inter-atomic potential energy model for finite and extended systems, in Proceedings of the 32nd International Conference on Neural Information Processing Systems, NIPS'18 (Curran Associates Inc., Red Hook, NY, USA, 2018) p. 4441-4451.

[64] H. Wang, L. Zhang, J. Han, and W. E, DeePMD-kit: A deep learning package for many-body potential energy representation and molecular dynamics, Comput. Phys. Commun. 228, 178 (2018).

[65] G. Kresse and F. J, Efficient iterative schemes for ab initio total-energy calculations using a plane-wave basis set, Phys. Rev. B 54, 11169 (1996).

[66] G. Kresse and J. Furthmüller, Efficiency of ab-initio total energy calculations for metals and semiconductors using a plane-wave basis set, Comput. Mater. Sci. 6, 15 (1996).

[67] P. E. Blochl, Projector augmented-wave method, Phys. Rev. B 50, 17953 (1994).

[68] G. Kresse and D. Joubert, From ultrasoft pseudopotentials to the projector augmented-wave method, Phys. Rev. B 59, 1758 (1999).

[69] J. P. Perdew, K. Burke, and M. Ernzerhof, Generalized gradient approximation made simple, Phys. Rev. Lett. 77, 3865 (1996).

[70] M. P. Allen and D. J. Tildesley, Computer Simulation of Liquids (Oxford University Press, 2017). 
[71] S. Plimpton, Fast parallel algorithms for short-range molecular dynamics, J. Comput. Phys. 117, 1 (1995).

[72] A. Togo and I. Tanaka, First principles phonon calculations in materials science, Scr. Mater. 108, $1(2015)$.

[73] A. R. Oganov and C. W. Glass, Crystal structure prediction using ab initio evolutionary techniques: Principles and applications, J. Chem. Phys. 124, 244704 (2006).

[74] A. O. Lyakhov, A. R. Oganov, H. T. Stokes, and Q. Zhu, New developments in evolutionary structure prediction algorithm USPEX, Comput. Phys. Commun. 184, 1172 (2013).

[75] A. R. Oganov, A. O. Lyakhov, and M. Valle, How evolutionary crystal structure prediction works - and why, Acc. Chem. Res. 44, 227 (2011).

[76] https://github.com/sliutheorygroup/structure/tree/main/l17-in ${ }_{2} \mathrm{se}_{3}$.

[77] J. Liu and S. T. Pantelides, Pyroelectric response and temperature-induced $\alpha-\beta$ phase transitions in $\alpha$ - $\mathrm{In}_{2} \mathrm{Se}_{3}$ and other $\alpha-\mathrm{III}_{2} \mathrm{VI}_{3}(\mathrm{III}=\mathrm{Al}, \mathrm{Ga}$, In; VI= S, Se) monolayers, 2D Mater. 6, 025001 (2019).

[78] Y. Qi, S. Liu, I. Grinberg, and A. M. Rappe, Atomistic description for temperature-driven phase transitions in $\mathrm{BaTiO}_{3}$, Phys. Rev. B 94, 134308 (2016).

[79] R. B. Wexler, Y. Qi, and A. M. Rappe, Sr-induced dipole scatter in $\mathrm{Ba}_{\mathrm{x}} \mathrm{Sr}_{1-\mathrm{x}} \mathrm{TiO}_{3}$ : Insights from a transferable-bond valence-based interatomic potential, Phys. Rev. B 100, 174109 (2019).

[80] P. J. Steinhardt, D. R. Nelson, and M. Ronchetti, Bond-orientational order in liquids and glasses, Phys. Rev. B 28, 784 (1983).

[81] W. Mickel, S. C. Kapfer, G. E. Schröder-Turk, and K. Mecke, Shortcomings of the bond orientational order parameters for the analysis of disordered particulate matter, J. Chem. Phys. 138, 044501 (2013).

[82] http://dplibrary.deepmd.net.

[83] J. Sun, A. Ruzsinszky, and J. P. Perdew, Strongly constrained and appropriately normed semilocal density functional, Phys. Rev. Lett. 115, 036402 (2015).

[84] E. B. Isaacs and C. Wolverton, Performance of the strongly constrained and appropriately normed density functional for solid-state materials, Phys. Rev. Materials 2, 63801 (2018).

[85] R. B. Wexler, G. S. Gautam, and E. A. Carter, Exchange-correlation functional challenges in modeling quaternary chalcogenides, Phys. Rev. B 102, 054101 (2020). 
TABLE I. DFT and DP energy $(E)$ in eV per 5-atom unit cell for monolayer $\operatorname{In}_{2} \mathrm{Se}_{3}$ polymorphs in reference to monolayer $\alpha-\operatorname{In}_{2} \mathrm{Se}_{3}$. Lattice constants $a$ and $b$ in $\AA$ are optimized with DFT. The $\alpha, \beta, \beta^{\prime}$ phases have hexagonal unit cells while $\beta_{1}^{\prime}, \beta_{2}^{\prime}, \beta_{3}^{\prime}$ have orthorhombic unit cells.

\begin{tabular}{lcccccc}
\hline \hline & $\alpha$ & $\beta_{3}^{\prime}$ & $\beta_{1}^{\prime}$ & $\beta^{\prime}$ & $\beta_{2}^{\prime}$ & $\beta$ \\
\hline$a$ & 4.063 & 8.093 & 4.093 & 3.963 & 3.962 & 3.982 \\
$b$ & 4.063 & 7.045 & 6.816 & 4.040 & 6.816 & 3.982 \\
$E_{\mathrm{DFT}}$ & 0 & 0.0308 & 0.0483 & 0.0523 & 0.0527 & 0.1485 \\
$E_{\mathrm{DP}}$ & 0 & 0.0157 & 0.0461 & 0.0521 & 0.0525 & 0.1703 \\
\hline \hline
\end{tabular}

TABLE II. Steinhardt's bond orientational order parameters $\left(Q_{l}\right)$ for Se atoms in monolayer $\operatorname{In}_{2} \mathrm{Se}_{3}$ of $\alpha$ and $\beta$ phases. The $\alpha$ phase has polarization pointing downward as shown in Fig. 1 .

\begin{tabular}{lllllll}
\hline \hline & $Q_{4}^{\text {top }}$ & $Q_{4}^{\text {mid }}$ & $Q_{4}^{\text {bottom }}$ & $Q_{6}^{\text {top }}$ & $Q_{6}^{\text {mid }}$ & $Q_{4}^{\text {bottom }}$ \\
\hline$\alpha$ & 0.073 & 0.049 & 0.141 & 0.163 & 0.175 & 0.245 \\
$\beta$ & 0.116 & 0.082 & 0.082 & 0.101 & 0.166 & 0.166 \\
\hline \hline
\end{tabular}


(a)

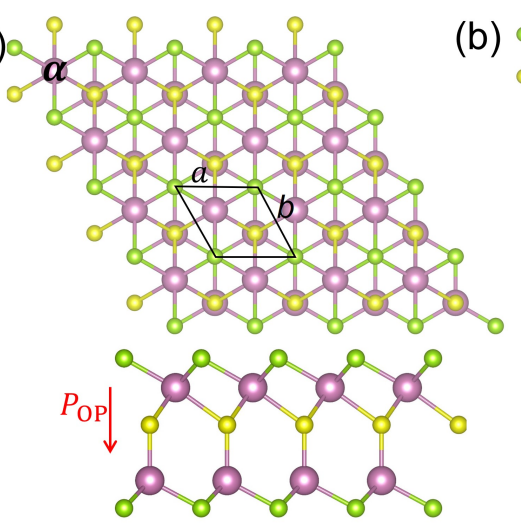

(d)
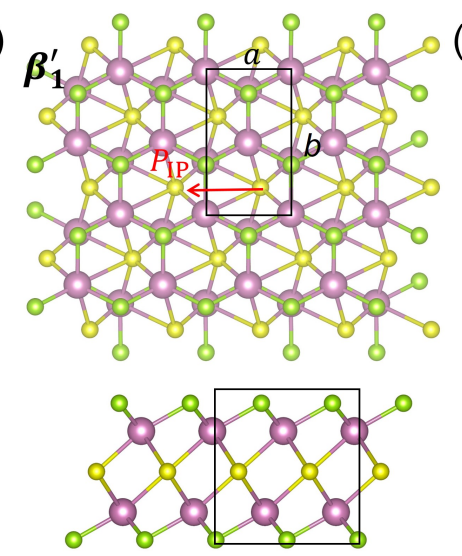

(b)

(e)

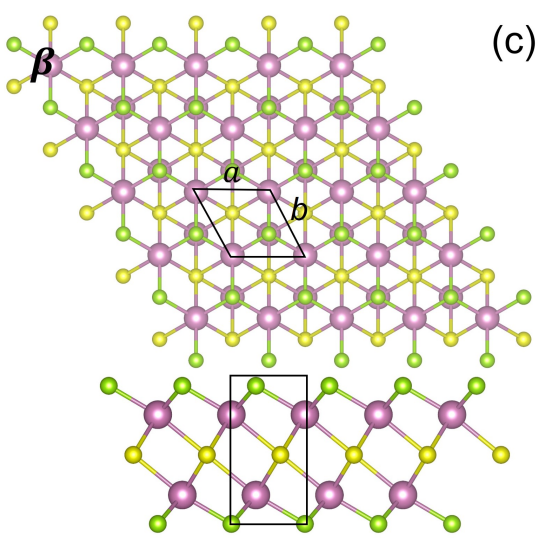

(c)
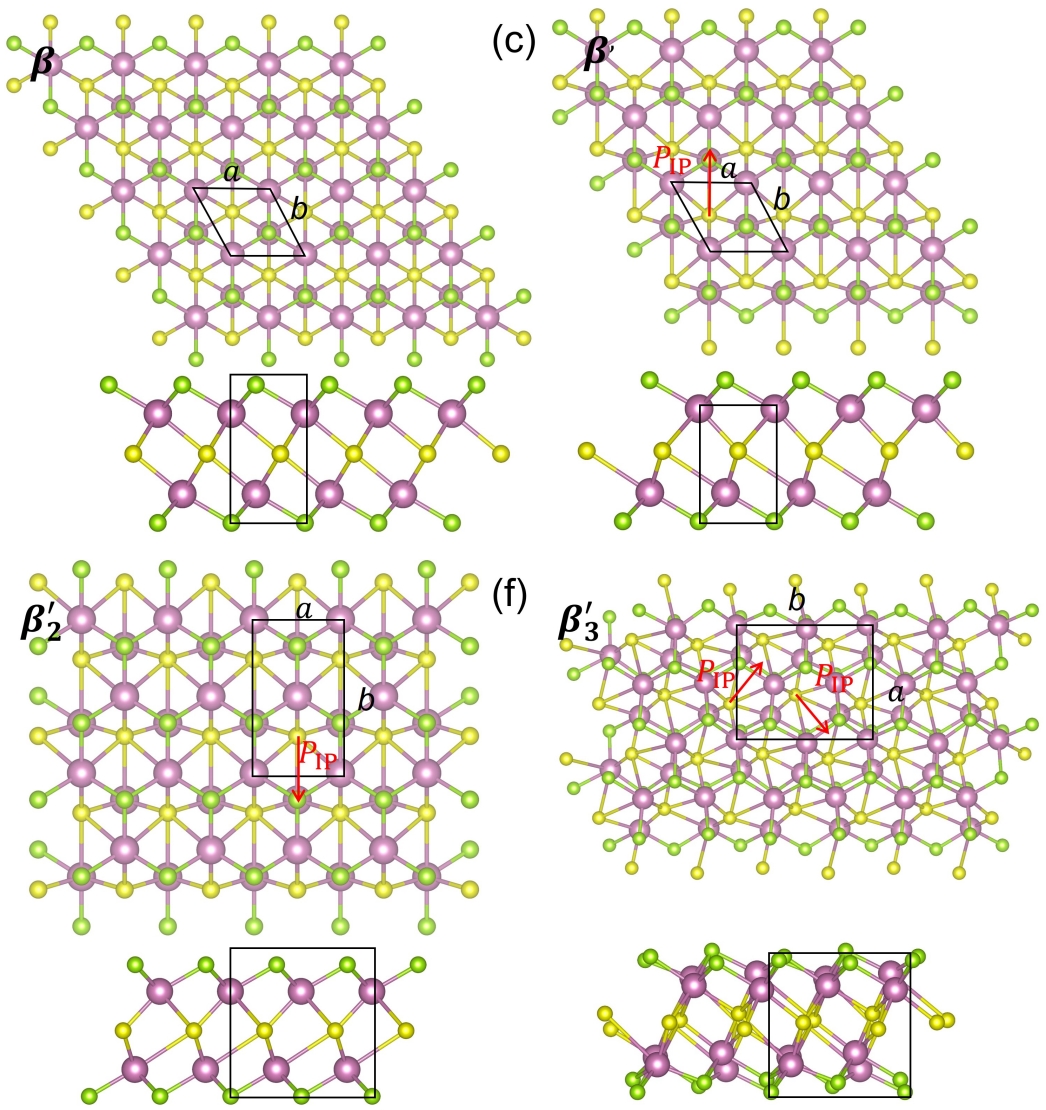

FIG. 1. Top and side views of (a) $\alpha$, (b) $\beta$, (c) $\beta^{\prime}$, (d) $\beta_{1}^{\prime}$, (e) $\beta_{2}^{\prime}$, and (f) $\beta_{3}^{\prime}$ phases of monolayer $\mathrm{In}_{2} \mathrm{Se}_{3}$. The Se atoms in the middle sublayer are colored in yellow while the Se atoms in the top and bottom sublayers are colored in green. The red arrow represents the direction of local polarization. 


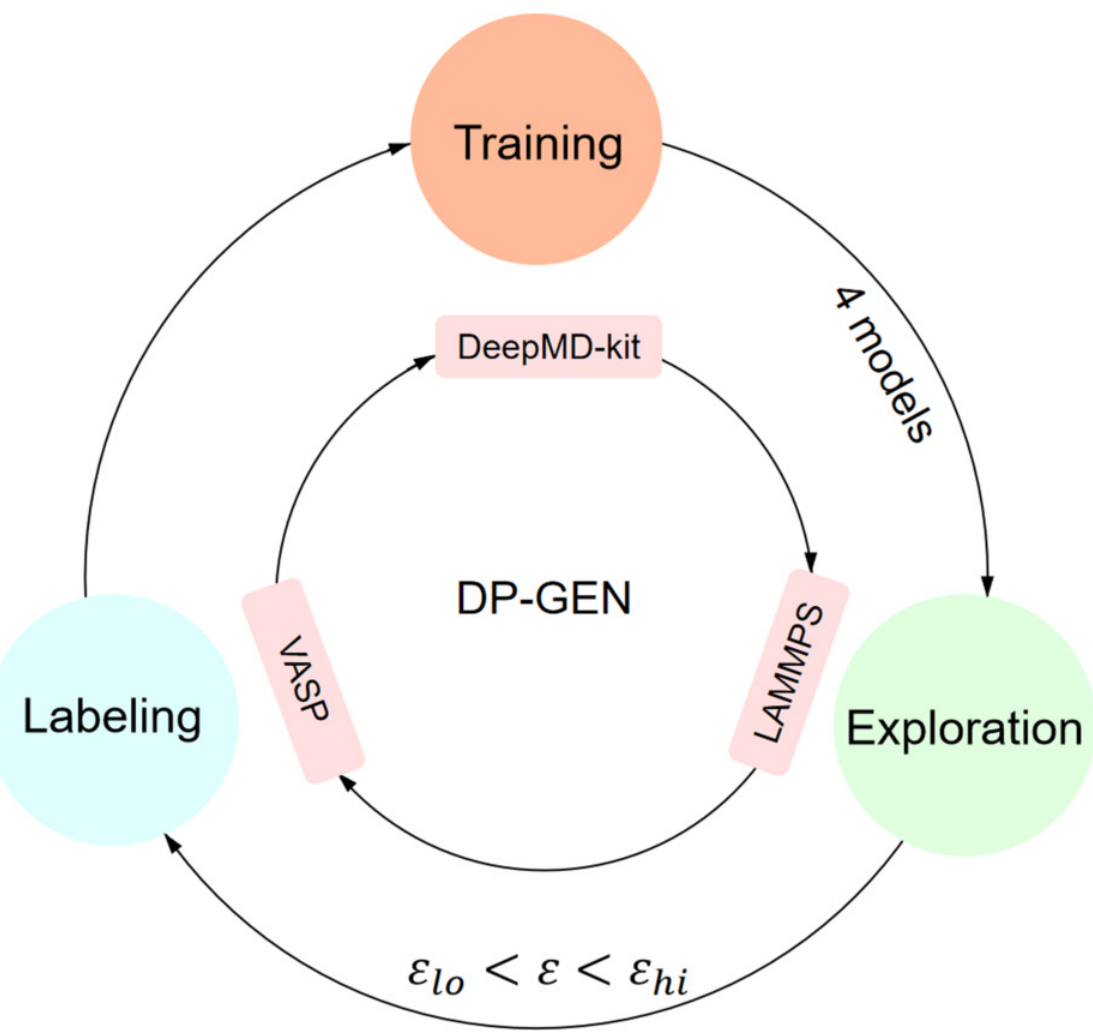

FIG. 2. (a) DP-GEN workflow with training, exploration, and labeling steps forming a closed loop. At the training step, four models are trained using first-principles energies, atomic forces, and stress. The model deviation $\mathcal{E}$ is used as an error indicator for importance sampling, and only configurations satisfying $\mathcal{E}_{\text {lo }}<\mathcal{E}<\mathcal{E}_{\text {hi }}$ are labeled for DFT calculations. 

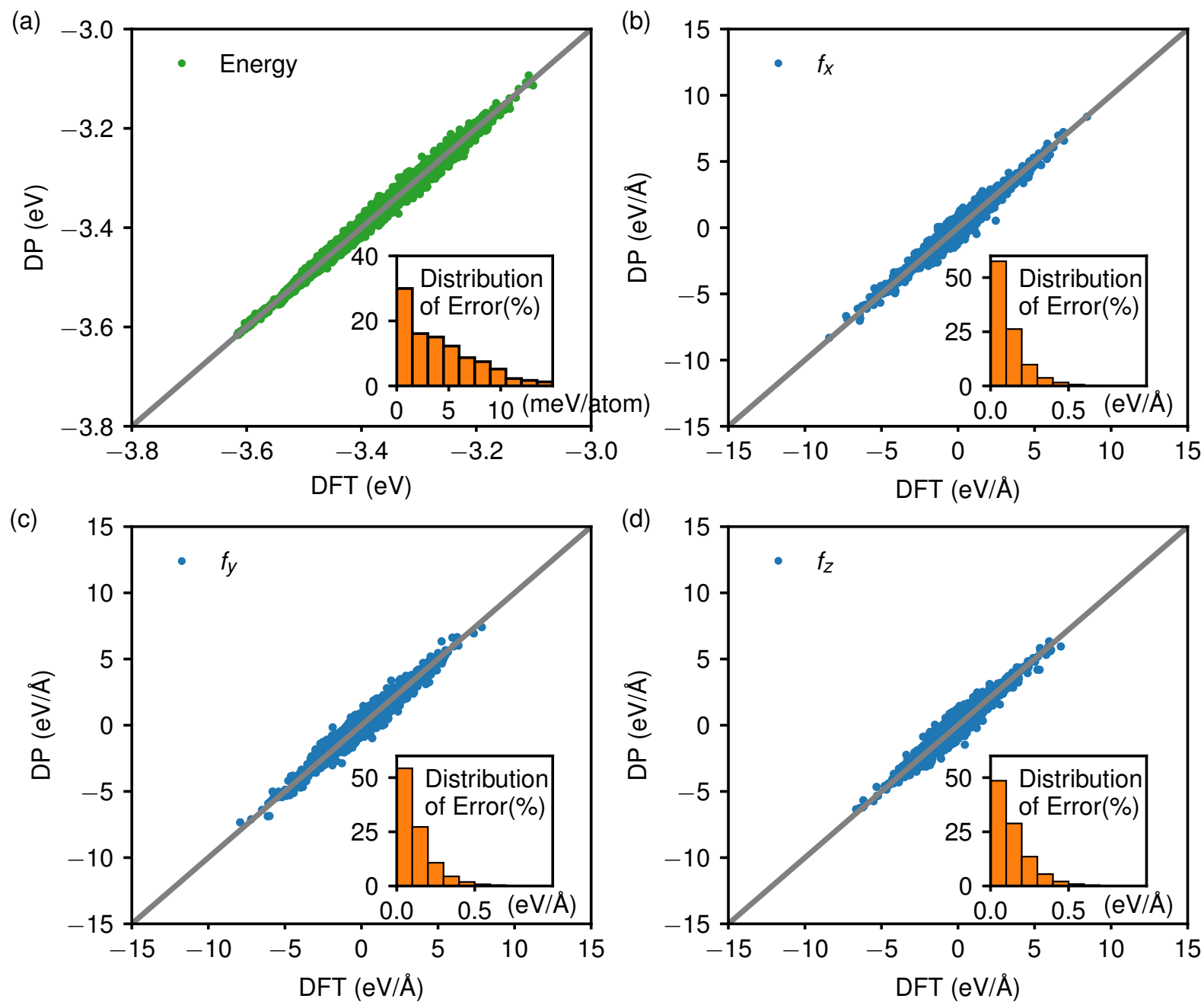

FIG. 3. Comparison of DFT and DP (a) energy per atom and (b-d) atomic forces for configurations in the final training database. The insets provide the distribution of the absolute error. 

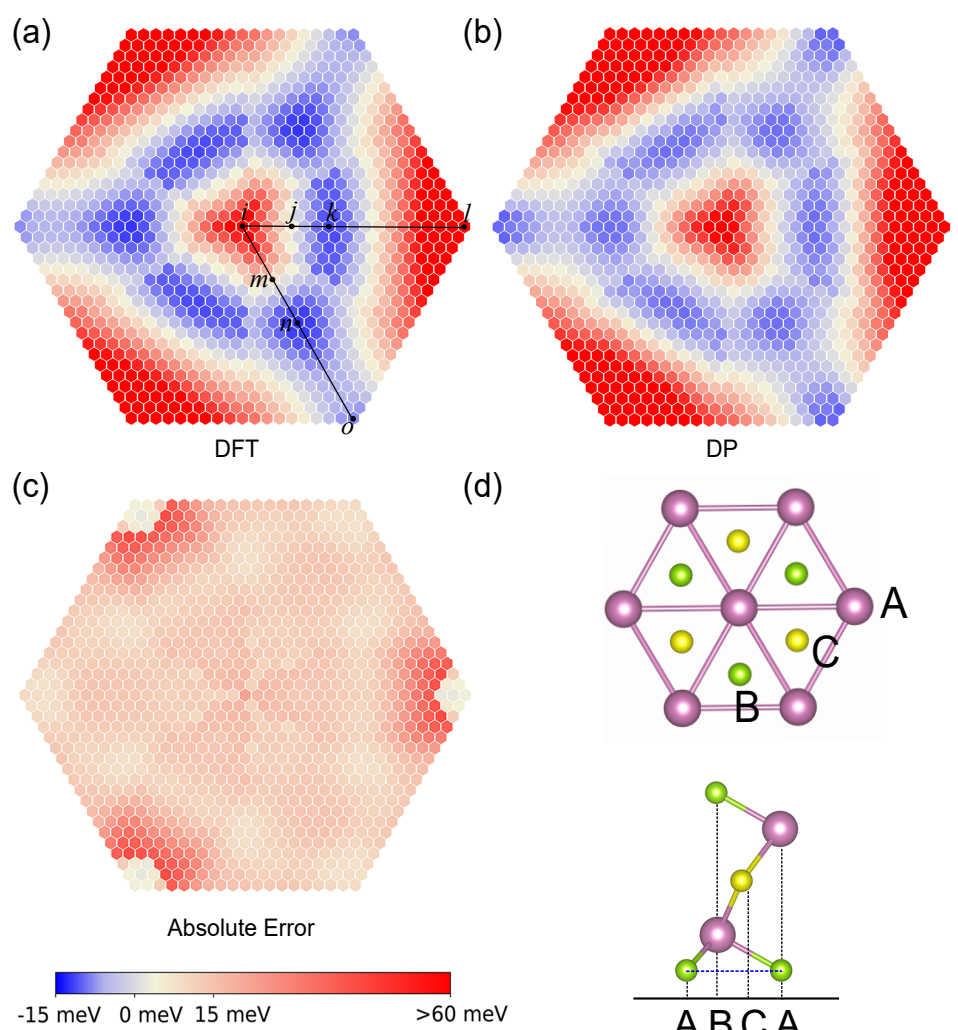

(e)
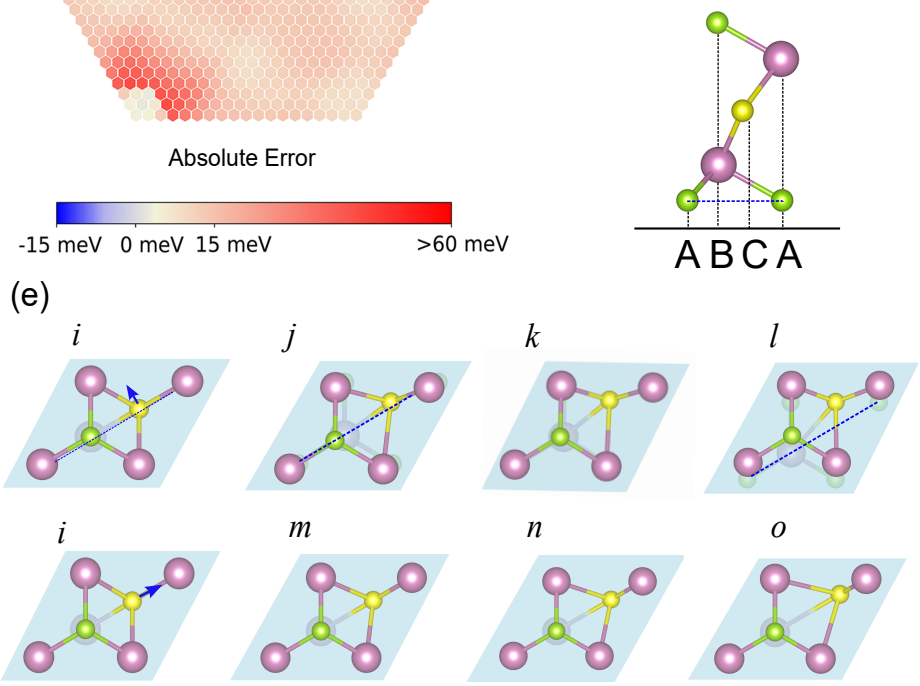

$n$

$O$
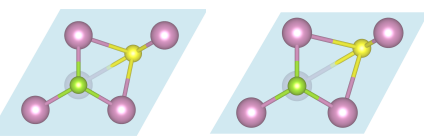

FIG. 4. Two dimensional potential energy surface for the in-plane sliding of the central Se sublayer in the QL computed with (a) DFT and (b) DP. As the structure has six-fold symmetry, the energy map was obtained by calculating the energies of configurations in the one sixth of the whole map area. (c) Absolute error in energy per 5-atom unit cell. (d) Top and side view of the QL used as the zero energy reference (denoted as fcc' in ref. 16). Atoms in each sublayer are arranged in one of the triangular lattices denoted as A, B, and C, respectively. (e) Configurations along two pathways highlighted in (a). For all the configurations, the central Se atom is fixed while all the other atoms are fully relaxed. 


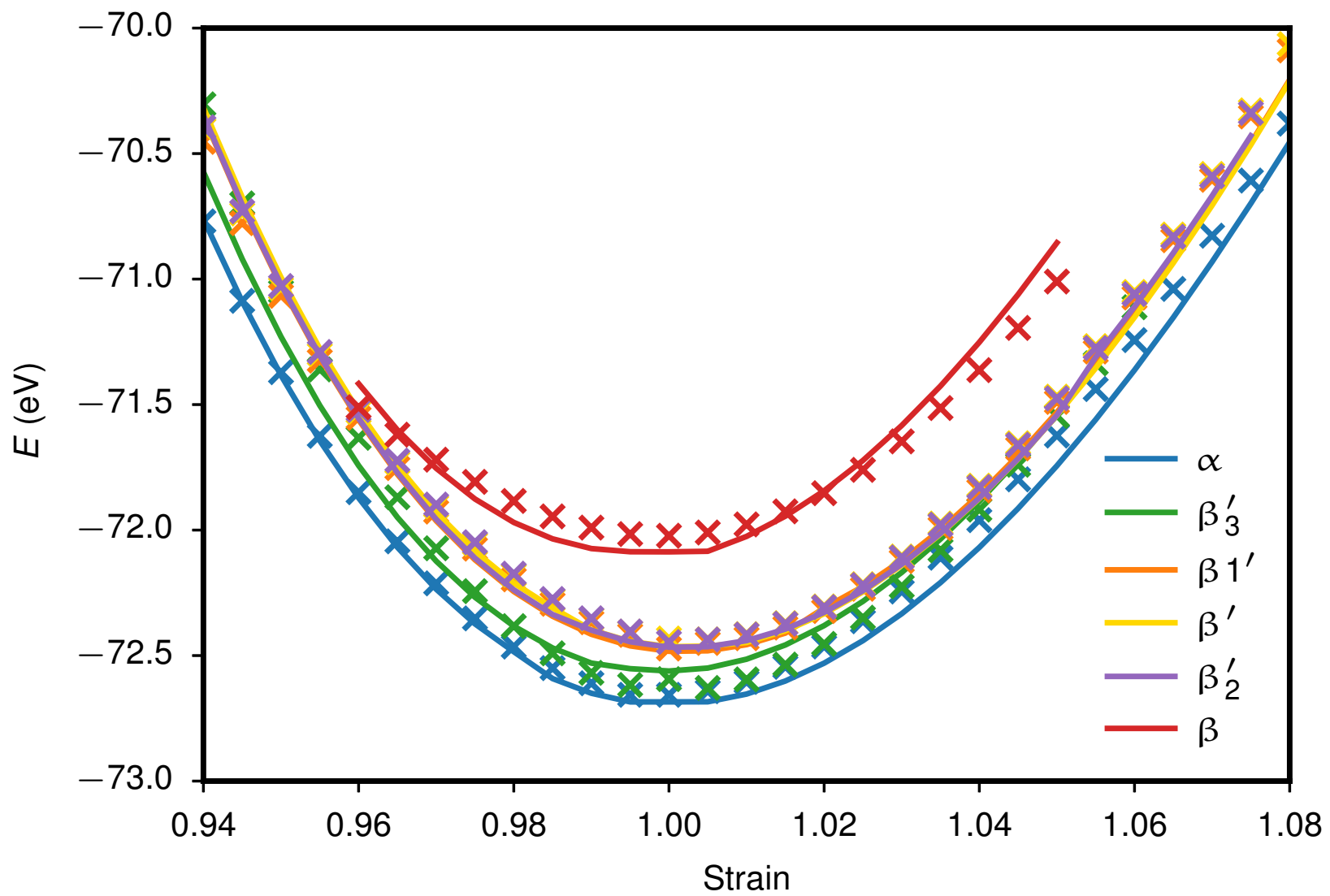

FIG. 5. Energy-strain plots for monolayer $\mathrm{In}_{2} \mathrm{Se}_{3}$ of different phases subjected to equibiaxial deformations. Solid lines and cross points denote DFT and DP results, respectively. 


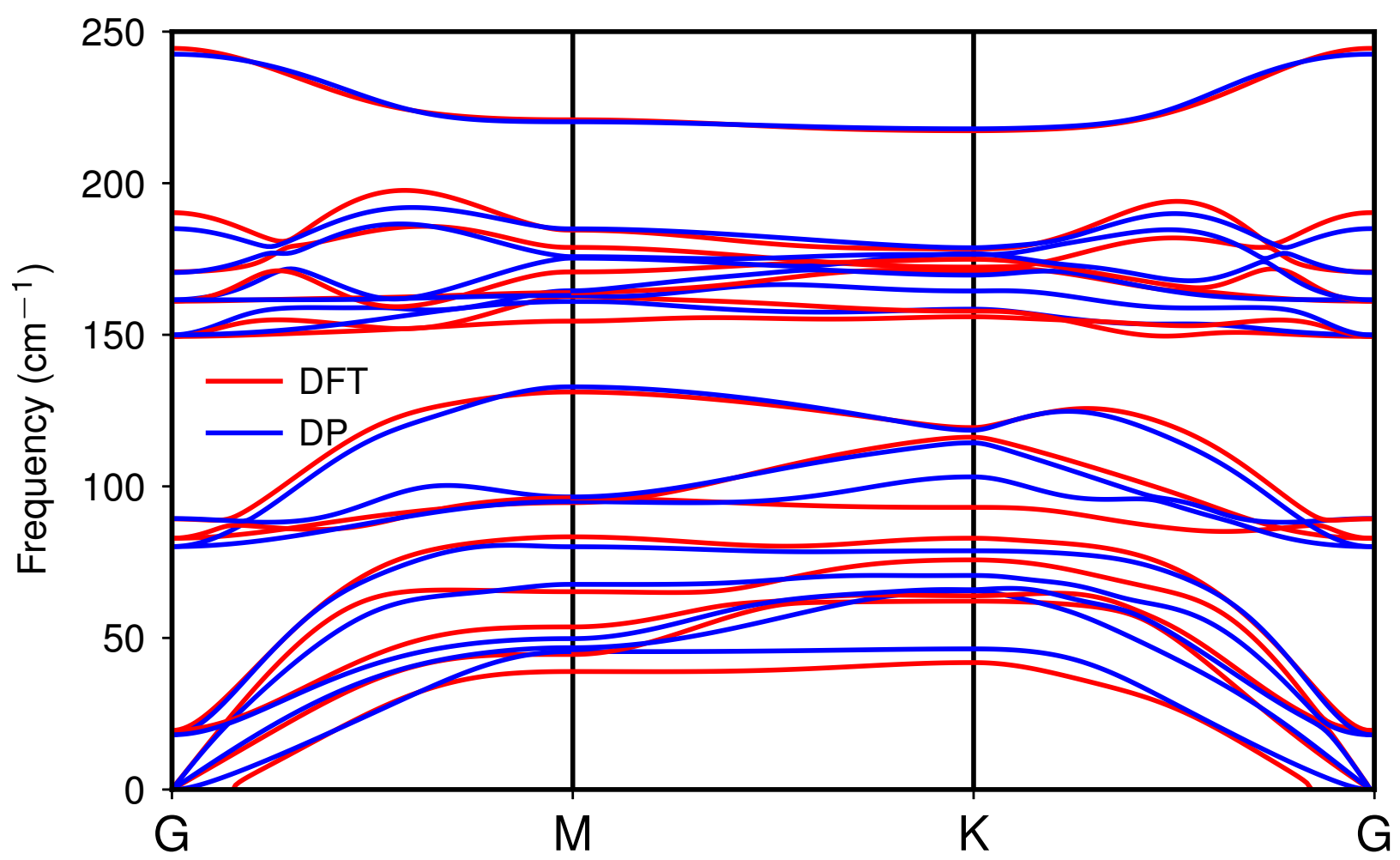

FIG. 6. Phonon dispersion relations of monolayer $\alpha-\mathrm{In}_{2} \mathrm{Se}_{3}$ computed with DFT and DP. 
(a)

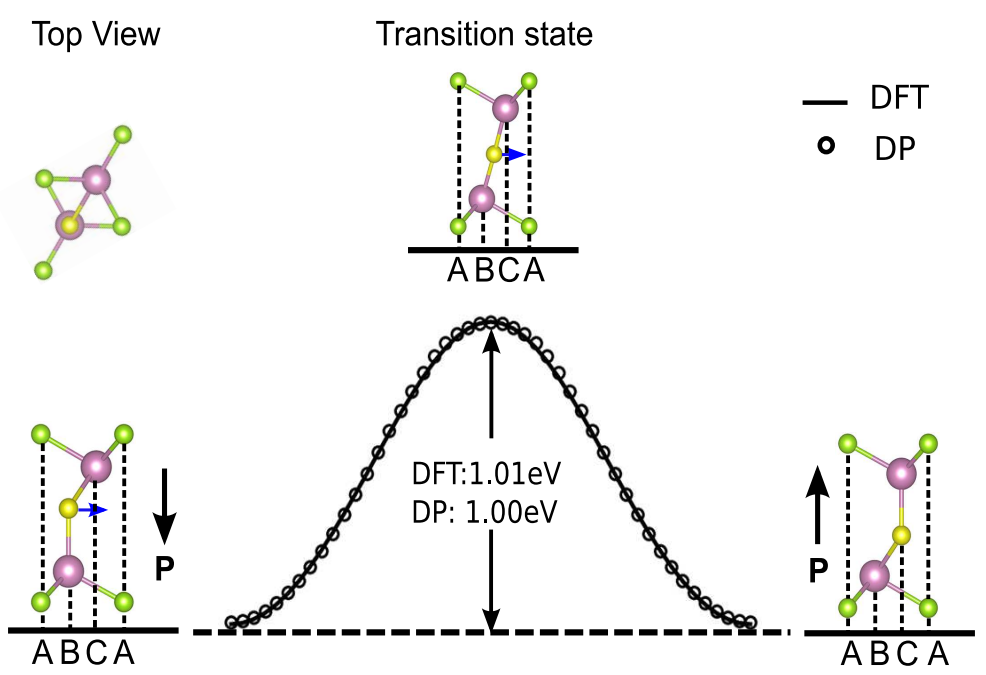

(b)

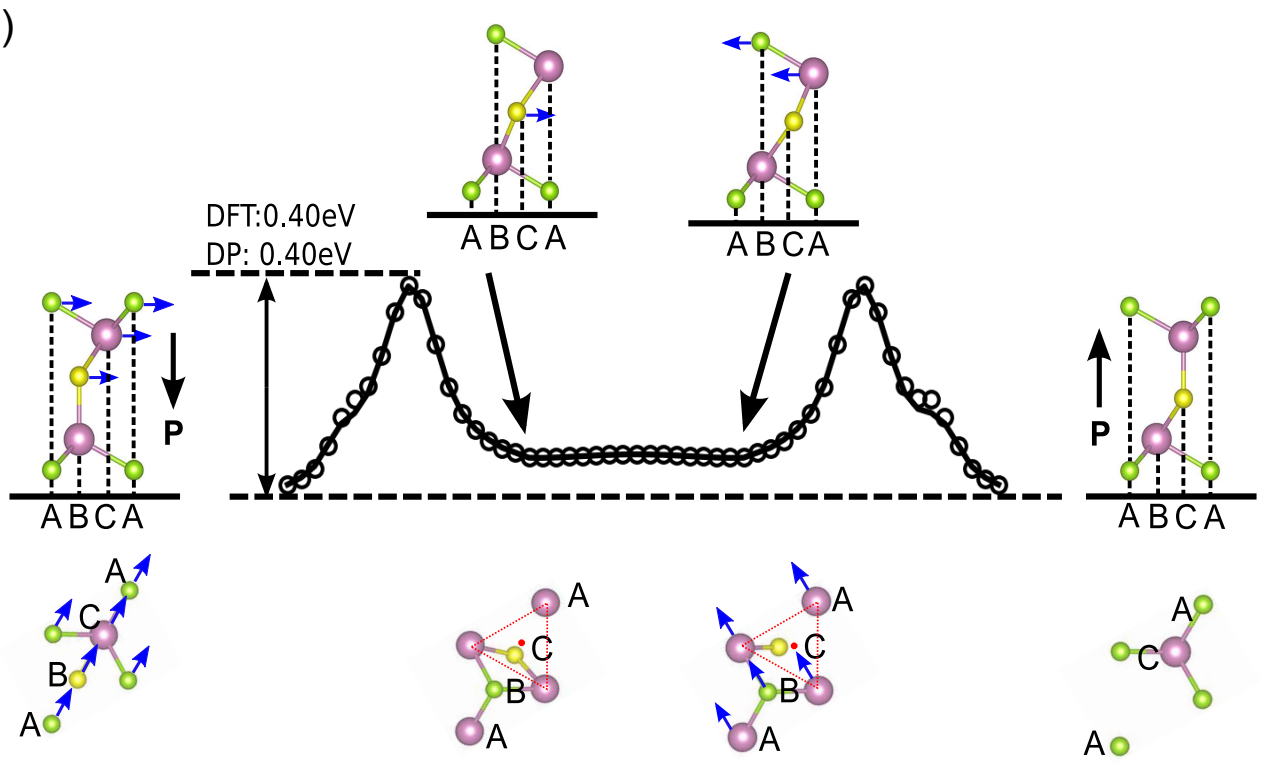

FIG. 7. DFT and DP energy profiles for polarization reversal pathways in monolayer $\alpha-\operatorname{In}_{2} \mathrm{Se}_{3}$. (a) Direct switching of out-of-plane polarization via the displacement of the central Se sublayer. (b) Multistep switching via concerted motions of upper Se-In-Se layers. Only the top three sublayers, Se-In-Se, are shown in the top view. The flat region of the energy profile corresponds to the rotation of the middle Se sublayer around the ideal C point (red dot) by $60^{\circ}$. 
(a)

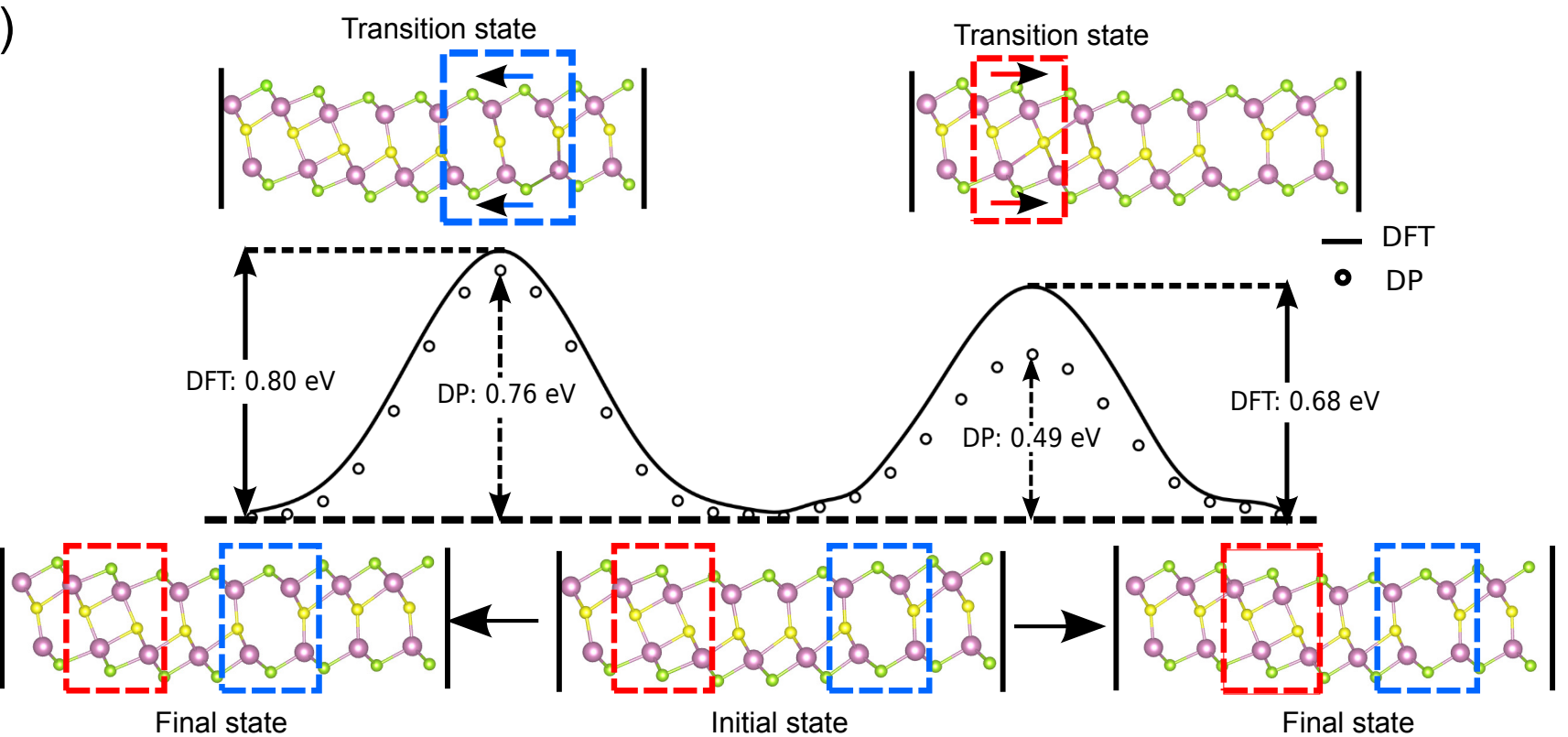

(b)
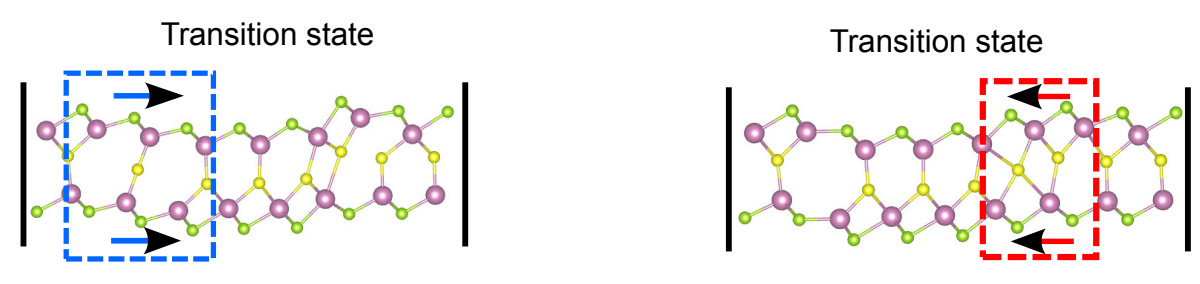

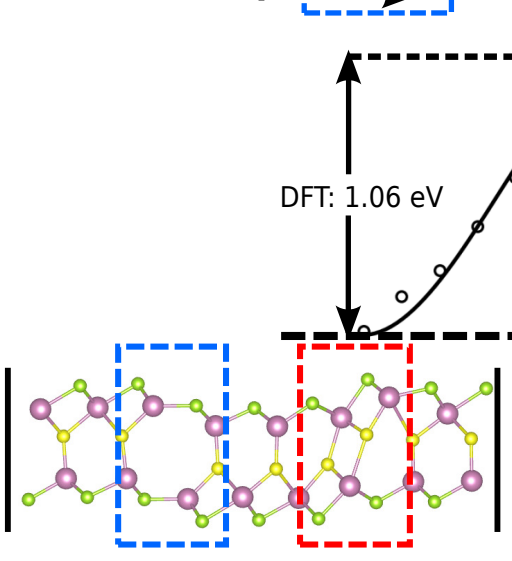

Final state

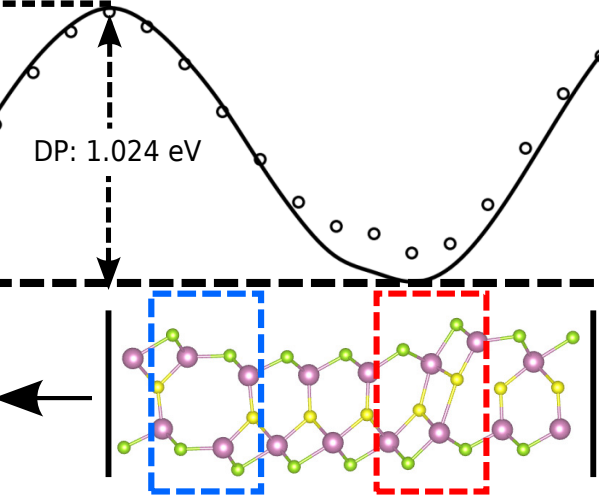

Initial state
Transition state
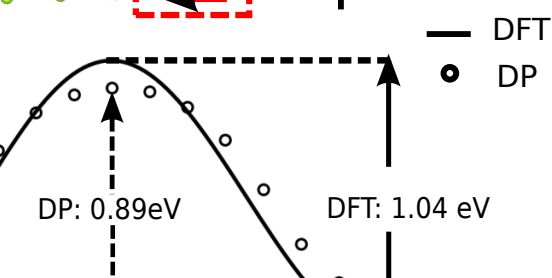

FIG. 8. DFT and DP energy profiles for $180^{\circ}$ domain wall motions in monolayer $\alpha-\operatorname{In}_{2} \mathrm{Se}_{3}$. Because of the in-plane lattice asymmetry, there are four different types of $180^{\circ}$ domain walls. The initial pathways were taken from ref. 16 and were re-optimized using a higher planewave cutoff energy and more intermediate images. 
(a)

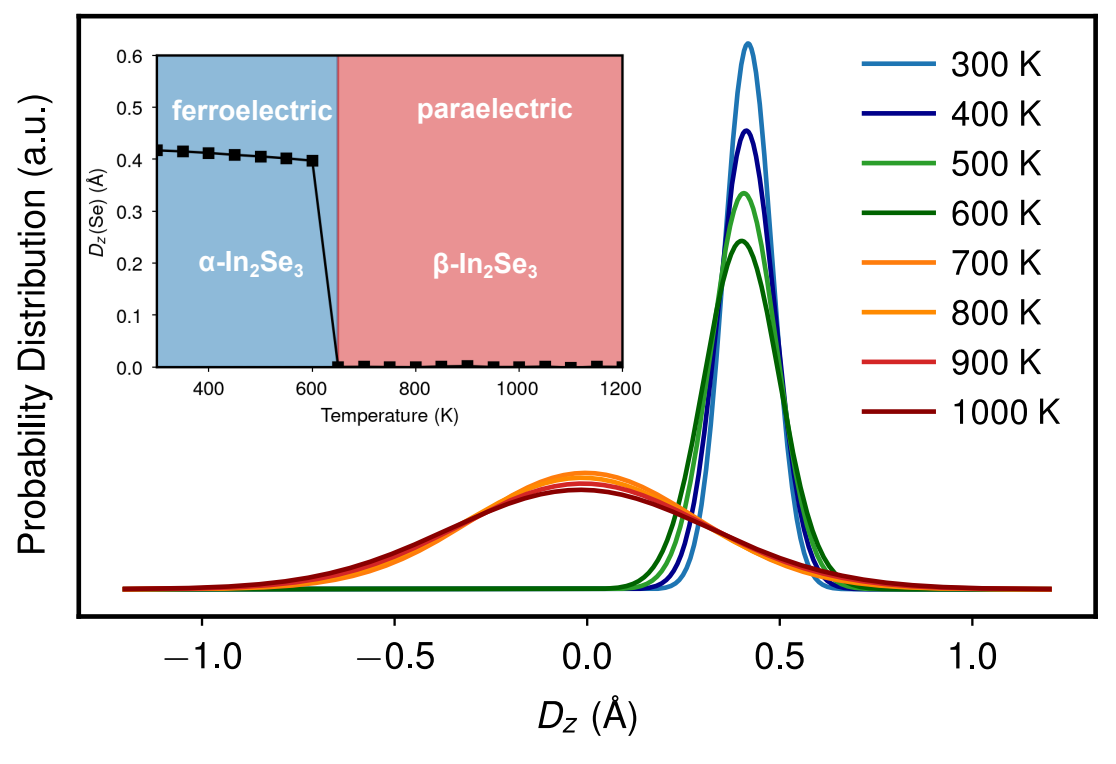

(b)
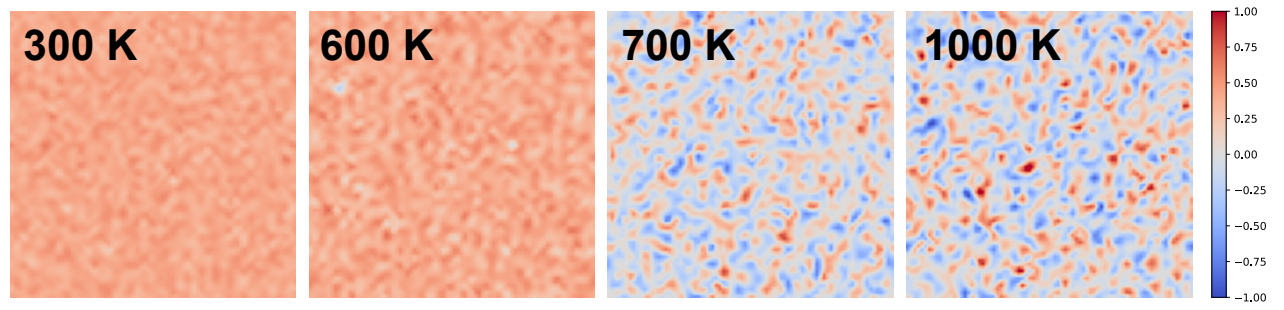

FIG. 9. Temperature-driven ferroelectric-paraelectric phase transition in monolayer $\alpha$ - $\operatorname{In}_{2} \mathrm{Se}_{3}$. (a) Temperature-dependent probability distributions of atomic displacements $\left(D_{z}\right)$ of central Se sublayer as a function of temperature. The inset shows the evolution of $D_{z}$ with increasing temperature, confirming a $\alpha \rightarrow \beta$ transition at $650 \mathrm{~K}$. (b) $D_{z}$ maps for instantaneous configurations sampled at different temperatures. 
(a)
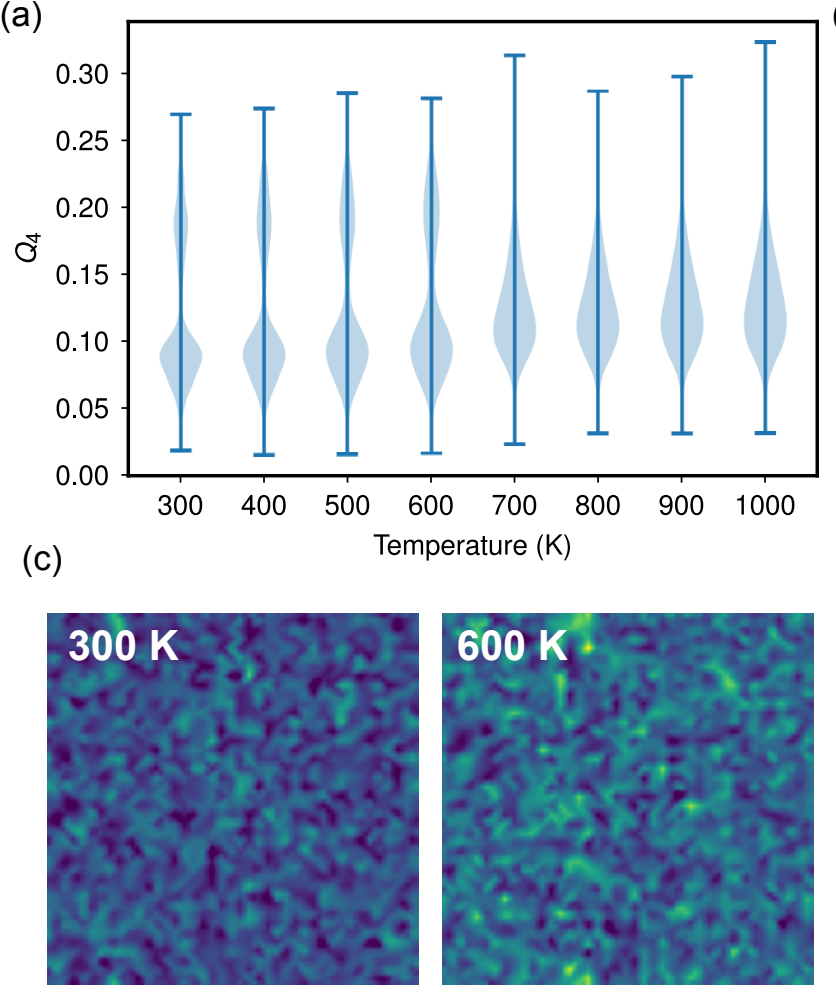

(b)
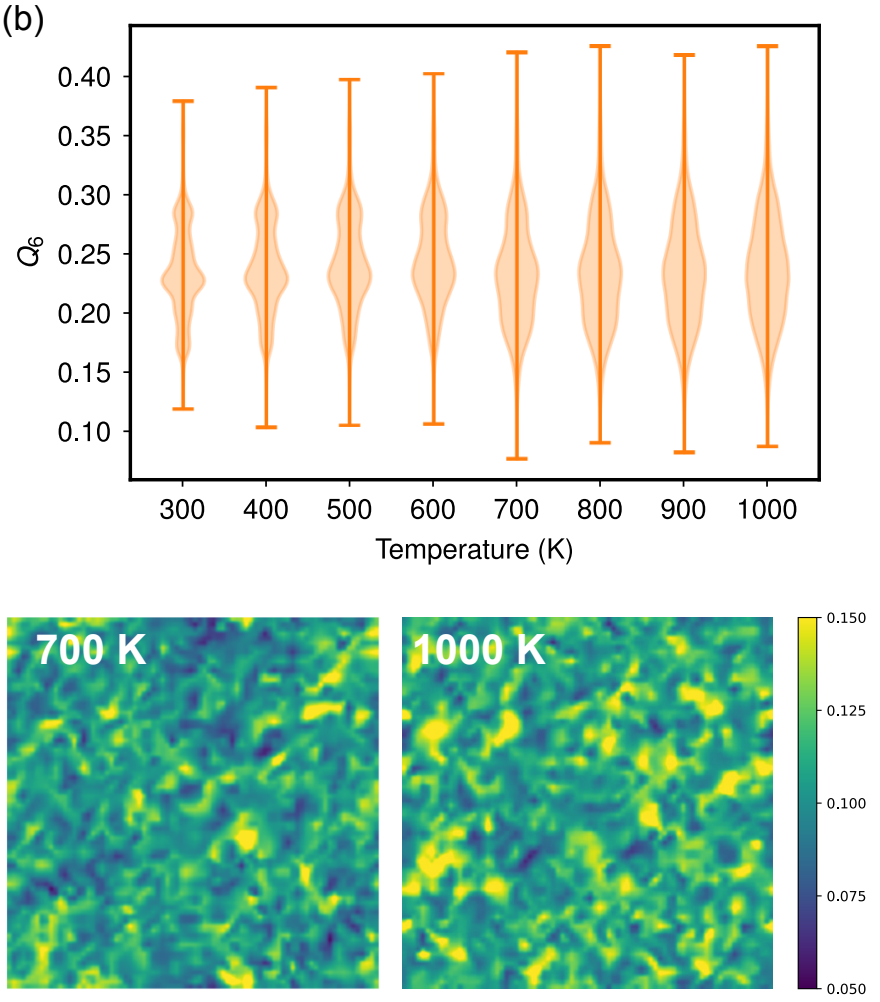

FIG. 10. Violin plot of (a) $Q_{4}$ and (b) $Q_{6}$ of Se atoms in monolayer $\alpha-\mathrm{In}_{2} \mathrm{Se}_{3}$ as a function of temperature. (c) 2D map of $Q_{4}$ for Se atoms in the middle sublayer for instantaneous configurations sampled at different temperatures. 